\title{
A single-cell atlas of non-parenchymal brain macrophages reveals unique transcriptional identities that are shaped by ontogeny and tissue environment.
}

Hannah Van Hove1,2, Liesbet Martens ${ }^{3,4}$, Karen De Vlaminck ${ }^{1,2}$, Ana Rita Antunes ${ }^{1,2}$, Sofie De Prijck ${ }^{4,5}$, Niels Vandamme $3,4,11$, Sebastiaan De Schepper6, Isabelle Scheyltjens ${ }^{1,2}$, Gert Van Isterdael7,8, Charlotte L. Scott $4,5,9$, Jeroen Aerts ${ }^{10}$, Geert Berx ${ }^{11,12}$, Guy E. Boeckxstaens 6 , Roosmarijn E. Vandenbroucke4,13,15, Lars Vereecke14,15,16, Diederik Moechars ${ }^{10}$, Martin Guilliams ${ }^{4,5}$, Jo A. Van Ginderachter1,2,\#, Yvan Saeys 3,17,\#, Kiavash Movahedi1,2,"

\footnotetext{
1 Myeloid Cell Immunology Lab, VIB Center for Inflammation Research, Brussels, Belgium

${ }^{2}$ Lab of Cellular and Molecular Immunology, Vrije Universiteit Brussel, Brussels, Belgium

3 Data Mining and Modeling for Biomedicine, VIB Center for Inflammation Research, Ghent, Belgium

${ }^{4}$ Department of Biomedical Molecular Biology, Ghent University, Ghent, Belgium

${ }^{5}$ Laboratory of Myeloid Cell Ontogeny and Functional Specialization, VIB Center for Inflammation Research, Ghent, Belgium Immunoregulation and Mucosal Immunology, VIB Center for Inflammation Research, Ghent, Belgium
}

6 Department of Chronic Diseases, Metabolism and Ageing, Translational Research Center for Gastrointestinal Disorders (TARGID), Intestinal Neuro-immune Interactions, University of Leuven, Leuven, Belgium

${ }^{7}$ Lab of Immunoregulation, VIB Inflammation Research Center, Ghent University, Ghent, Belgium

8 Department of Respiratory Medicine, University Hospital Ghent, Ghent, Belgium

9 Institute of Infection, Immunity and Inflammation, College of Medical, Veterinary and Life Sciences, University of Glasgow, UK.

10 Department of Neuroscience, Janssen Research \& Development, a Division of Janssen Pharmaceutica NV., Beerse, Belgium.

${ }^{11}$ Molecular and Cellular Oncology Laboratory, Department of Biomedical Molecular Biology, Ghent University, Ghent, Belgium

12 Cancer Research Institute Ghent (CRIG), Ghent, Belgium

${ }^{13}$ Barriers in Inflammation, VIB Center for Inflammation Research, Ghent, Belgium

14 Host-Microbiota Interaction lab (HMI), VIB Center for Inflammation Research, Ghent, Belgium

15 Ghent Gut Inflammation Group (GGIG), Ghent University, Ghent, Belgium

${ }^{16}$ Department of Rheumatology, University Hospital Ghent, Ghent, Belgium

17 Department of Applied Mathematics, Computer Science and Statistics, Ghent University, Ghent, Belgium

\# Equal contribution

* Correspondence: kiavash.movahedi@vub.vib.be 


\begin{abstract}
Increasing evidence suggests that macrophages critically shape brain homeostasis and disease. However, while the pivotal role of parenchymal microglia has gradually emerged, other brain-resident myeloid cells remain elusive. By dissecting border regions and combining single-cell RNA sequencing with high-dimensional cytometry, bulk RNA-sequencing, fate-mapping and microscopy, we reveal the remarkable diversity of non-parenchymal brain macrophages. Border-associated macrophages or BAMs residing in the dura mater, subdural meninges and choroid plexus consisted of distinct subsets that exhibited tissue-specific transcriptional signatures and underwent strong compositional changes during postnatal development. The gene regulatory networks of BAMs were identified and fundamentally differed from those of microglia. Importantly, we identified a unique non-homeostatic microglia-like population residing on the apical surface of the choroid plexus epithelium. Niche accessibility drove BAM ontogeny and determined whether embryonic macrophages were progressively replaced by bone marrow progenitors. Together, our work provides important insights into the biology of brain macrophages and offers a solid framework for future investigations.
\end{abstract}




\section{Introduction}

The brain is a compartmentalized organ that contains diverse border regions, which have important protective and regulatory functions and form the interface with the periphery. Each of these borders is associated with its own degree of steady-state "immune privilege" due to the presence of distinct endothelial and epithelial barriers ${ }^{1,2}$. The brain parenchyma is covered by the three-layered meninges. The upper layer contains the dura mater, which is attached to the skull and constitutes a more permissive border due to the presence of fenestrated blood vessels and lymphatics ${ }^{2,3}$. The dura is separated from the arachnoid and the pia mater by the arachnoid barrier epithelial cells, which contain tight junctions and limit accessibility to the underlying tissue. In contrast to the dural vessels, the vasculature in the subdural meninges contains tight junctions forming the blood-leptomeningeal barrier. Directly underneath the pia mater lies the glia limitans superficialis, a layer of astrocytic processes that block the entry of cells from the cerebrospinal fluid (CSF) into the brain parenchyma². The perivascular space around the parenchymal vasculature also forms a border region with low accessibility due to the presence of the blood-brain barrier2. Finally, the choroid plexus (CP), which floats in the brain's ventricles, contains fenestrated blood vessels that form a dense network embedded in the CP stroma ${ }^{1,2,4}$. This loose connective tissue is lined by a single layer of CP epithelial cells that are interconnected with tight junctions and form the blood-CSF barrier. Epithelial barriers are suggested to be more permissive and act as the main entry gate for immune cells under homeostatic conditions'. The brain has recently been shown to contain a heterogeneous immune compartment, with most of the diversity thought to be restricted to its border regions ${ }^{5-7}$. However, the immune cell heterogeneity in the brain's borders has not yet been directly mapped, since analyses have only been performed at the whole brain level.

Macrophages are professional phagocytes that are integral to innate immune defense but are also emerging as critical regulators of tissue homeostasis ${ }^{8}$. Tissue-resident macrophages are extremely diverse and highly adapted to the microenvironment in which they reside, exhibiting tissue-specific epigenetic landscapes and transcriptional profiles ${ }^{9-11}$. A clear example of a macrophage population that plays an instrumental role in tissue homeostasis are microglia, the tissue-resident macrophages of the brain parenchyma. Homeostatic microglia are highly dynamic cells that continuously survey neuronal synapses and contribute to activity-dependent refinement of neural circuits via synaptic pruning ${ }^{12}$. Microglia are also key actors in neuroinflammatory and neurodegenerative diseases ${ }^{12}$. Extensive profiling of the microglial transcriptome during homeostasis and disease has provided insights into microglial gene expression, transcriptional regulation, development and disease-associated functions ${ }^{12-17}$. The advent of single-cell transcriptomics has also begun to unveil microglial heterogeneity and disease-associated responses, such as the recent identification of a novel microglial subset observed under conditions of neurodegeneration ${ }^{18}$. While the critical role of microglia in brain homeostasis and disease is gradually being uncovered, much less is known about the other tissueresident macrophages of the brain, residing at the brain's borders. Indeed, border associated macrophages or BAMs can be found in the perivascular space, meninges and in the $\mathrm{CP}$, where besides stromal macrophages, the CP epithelium also contains an uncharacterized macrophage-like population 
called the Kolmer's epiplexus cell 5,12,19,20. In the perivascular space and subdural meninges BAMs have an embryonic origin, while CP BAMs exhibit a mixed ontogeny ${ }^{19}$. The ontogeny of dural BAMs is unclear and it is unknown whether stromal CP macrophages and Kolmer's epiplexus cells share a similar ontogeny. Besides a limited analysis on perivascular macrophages ${ }^{19}$, no transcriptional profiles exist for clearly identified BAM subsets and their functions and role in health and disease are unclear.

In order to uncover the full immune diversity at the brain's borders together with a detailed analysis of its macrophage populations, we isolated individual border regions and employed droplet-based singlecell RNA sequencing, combined with complementary approaches. This yielded extensive insights into the immune cell diversity in the brain's borders and revealed the tissue-specific signatures of BAMs, including the identification of a microglial-like subset lining the apical surface of the CP epithelium. In addition, different fate-mapping models and developmental analyses allowed us to refine the ontogeny of BAMs and to assess their post-natal maturation, which revealed remarkable age-dependent changes in their composition and phenotype. 


\section{Results}

Unraveling regional immune cell heterogeneity in the brain via single-cell RNA sequencing of discrete brain compartments.

To obtain a comprehensive understanding of immune cell heterogeneity in the homeostatic brain, we isolated whole mouse brains or micro-dissected its border regions for single-cell RNA sequencing (scRNA-Seq) analysis (Fig. 1A). The dura mater was collected from the skull cap. Since in mice we were unable to peel off the pia/arachnoid mater from the parenchyma, thin slices were collected from the dorsal surface of the brain to enrich for the subdural (SD) meninges. The choroid plexus (CP) was removed from the lateral and fourth ventricles, taking care to exclude parenchymal contamination. Single-cell suspensions were made and CD45+ hematopoietic cells were FACS sorted, followed by scRNA-Seq using the 10x chromium platform. We relied on the SCRAN and Scater workflow for gene normalization ${ }^{21}$ and Seurat ${ }^{22}$ for unsupervised clustering, dimensionality reduction and t-Distributed Stochastic Neighbour Embedding (tSNE) projections of 6530 cells from the whole brain (Fig. 1B), 6564 cells from the SD meninges (Fig. 1C), 5865 cells from the dura mater (Fig. 1D) and 3940 cells from the CP (Fig. 1E). Clusters were identified based on the expression of known marker genes (Supplementary Table 1). As expected, the majority of immune cells in homeostatic whole brains were microglia (Fig. 1B), which were easily identified based on a set of signature genes (see Fig. 2C). The brain also contained various lymphocyte populations, including subsets of $B, T, N K T$ and NK cells, which were found in varying numbers in the distinct brain regions (Fig. 1B-E). Importantly, the brain and in particular its borders, contained a large and heterogeneous myeloid compartment, which was investigated in more depth.

Dendritic cells (DCs) were detected in all samples, but were most prevalent in the dura mater, where multiple subsets were easily identified (Fig. 1D, clusters 5-8). All DC subsets expressed Flt3, Tnni2, H2-Oa and Kctd14 and were low for the macrophage markers Adgre1 and Fcgr1 (Fig. 1D, F). The dura contained a plasmacytoid DC (pDC) cluster, with high expression of known pDC signature genes, including Siglech, Ccr9 and Pacsin123. DCs could be further subdivided into the conventional type 1 (cDC1) and type 2 (cDC2) subsets ${ }^{24}$. In accordance with literature, $c D C 1 s$ were high for the lineagedefining transcription factors Iff8, Batf3 and $I d 2$ and expressed a plethora of unique signature genes, including Xcr1 (Fig. 1F). cDC2s were IRF8 Zeb2hi, expressed Itgam (CD11b) and Sirpa and we identified signature expression of Cd209a, Tnfsf9, Tnip3 and Kcne3 (Fig. 1F). Remarkably, we also observed a DC cluster with a gene signature reminiscent of migratory DCs ${ }^{23}$ (migDCs) (Fig. 1D cluster 8), including the expression of Ccr7, Nudt17, Socs2, Cacnb3, Adcy6 and Tmem123. While the dura mater contained the highest number of migDCs, they were also observed in the choroid plexus. Importantly, this suggests that the brain's borders contain migDCs, which may migrate to the draining lymph nodes. The ratio of the various DC subsets differed in the distinct border regions (Fig. 1G). CDC2s were the dominant subset in the pia and dura mater, while in the CP the majority were CDC1s. pDCs were mainly restricted to the dura mater.

The dura mater also contained the highest number of monocytes, including both classical and nonclassical subsets ${ }^{25}$, and a monocyte/macrophage intermediate population termed monocyte-derived 
cells (MdCs) (Fig. 1D). Interestingly, macrophages that were clearly distinct from microglia were observed in the whole brain sample and detected in large numbers in the dissected border regions (red clusters in Fig.1B-E). These Border Associated Macrophages or $\mathrm{BAMs}^{5}$, were identified based on: (1) a high expression of prototypical macrophage genes, including Csf1r, Cd68, Adgre1 (F4/80), Fcgr1 (CD64), Aif1 (IBA1) and Cx3cr1; (2) a low expression of monocyte genes such as Ly6c2 (classical monocytes and MdCs) or Ace (non-classical monocytes); and (3) a low expression of CDC genes (see dot plots Fig. 1C-E). Compared to microglia, most dura and CP BAMs expressed low levels of the macrophage marker Mertk, but were high for $A x l$, which is another TAM receptor tyrosine kinase 26 . While we expect the majority of BAMs in the enriched SDM sample to represent pial and arachnoid macrophages, a small fraction may be perivascular macrophages that are derived from the thin cortical region underneath the pia mater, which also contained "contaminating" microglia (Fig. 1C). In the CP substantial heterogeneity was observed at the level of immediate early genes (IEGs). The CP BAM A population was split into two major clusters (CP-BAM A1 and CP-BAM A2, Supplementary Fig. 1A, B), due to a differential expression of IEGs (Supplementary Fig. 1C), which was observed in independent experiments. A likely explanation is dissociation-induced IEG induction in part of the population, which has been previously reported for different cell types ${ }^{27,28}$. Since dissociation-induced IEGs could mask the inherent heterogeneity of CP BAMs, this source of potentially confounding variation was successfully regressed out of the data (Supplementary Fig. 1A, D, E).

Together, these data uncover the complexity of the brain's immune compartment, providing compelling evidence for the existence of many transcriptionally unique lymphoid and myeloid subsets that reside in varying amounts in the brain's borders.

\section{Border-associated macrophages are highly heterogeneous and exhibit tissue-specific transcriptional signatures that are driven by distinct gene regulatory networks.}

To further characterize BAM heterogeneity and potential tissue-specific signatures, microglia and BAMs from the whole brain or the distinct border regions were pooled in a single data set consisting of 10.947 brain macrophages, which was subjected to unsupervised clustering and tSNE projection (Fig. 2A). Interestingly, this revealed at least six major BAM subsets, which all clustered separate from microglia (Fig. 2A, B). Cluster SD-BAM mainly consisted of subdural macrophages. Dural macrophages were split into two major subsets: Dlo-BAMs and Dhi-BAMs (nomenclature refers to low and high MHC II expression, see below). A small subset of subdural macrophages (representing cluster SD-BAM B in Fig. 1C) co-clustered with dural macrophages in Dhi-BAM. CP macrophages clustered separate from meningeal macrophages and most were found in clusters CPlo-BAM and CPhi-BAM (nomenclature refers to low and high MHC II expression, see below). Remarkably, a small subset of CP macrophages (representing cluster CP-BAM B in Fig. 1E) clustered separate from other BAMs or microglia (CPepiBAM, nomenclature refers to epiplexus, see Fig. 6). As the focus of this manuscript was on BAMs, we did not assess potential heterogeneity within the parenchymal microglia of young homeostatic mice and these cells were treated as a single population. 
Next, we examined the expression of previously reported microglial signature genes ${ }^{13,17}$ in the various BAM subsets and in classical monocytes, which served as a reference population (Fig. $2 \mathrm{C}$ ). While these signature genes were highly expressed in microglia, many were co-expressed to some degree in BAMs. Microglial signature genes that showed high to intermediate levels of expression in BAMs included Fcrls, Trem2 and Hexb. Others such as Olfm/3, Gpr34 and Tmem119 were co-expressed in some BAM subsets, albeit at low levels. Genes that were highly restricted to microglia included Sall1, Adgrg1, SIc2a5, Serpine2, Sparc and Adamts1. Interestingly however, in contrast to the other BAM subsets, CPepi-BAMs also expressed all microglial signature genes. While for most of these genes, their expression was lower than in microglia, others were non-differential (e.g. Fcrls, Trem2, Olfm/3, Siglech, Serpine2, Sparc). This suggests that while these CP macrophages were not identical to parenchymal microglia, they may have a shared ontogeny or phenotype.

BAMs exhibited distinct transcriptional states and we identified a set of signature genes that were either shared between the various BAM subsets or were unique for a specific population (Fig. 2D, E). In homeostatic conditions, Apoe was high in all BAMs, while low in parenchymal microglia. Other universal BAM signature genes included Ms4a7, Ms4a6c, Lyz2, Clec4a1, Ifitm2 and Tgfbi. Most of these universal BAM genes were absent or low in CPepi-BAMs, which more closely resembled the expression pattern of microglia. In all border regions, BAMs fell into two main groups, which we called $\mathrm{MHC} \mathrm{Il}^{\mathrm{lo}}$ and MHC IIhi BAMs. Notable genes that were differential between these groups included Mrc1, Cd163, Stab1, Igf1, Gas6 and Cd38, which were enriched in the MHC Illo group (SD-BAM, D'o-BAM, CPlo-BAM), while genes such as H2-Aa, H2-Eb1, H2-Dmb1, Cd74, Fxyd5, Cd72 and KIra2 were enriched in the MHC Il hi $^{\text {houp }}$ (Dhi-BAM and CPhi-BAM). However, BAMs also exhibited clear tissue-specific signatures. Genes that were enriched in meningeal MHC Il॰ BAMs (SD-BAM and D'o-BAM), included Clec4n, Clec10a, Cd209f and Folr2. SD-BAM were enriched for i.a. Lyve1, Ednrb, P2rx7 and Egfl7. Signature genes were also identified for the individual dural and CP BAM subsets. Examples include Ccl8 in Dlo-BAMs and Ttr in CP BAMs. CPepi-BAMs also exhibited a unique transcriptional profile, which included signature expression of Cst7, Mir155hg, Gm1673 and Maff.

In the dura and CP, genes that were differential between MHC IIlo and MHC II hi BAs often showed a graded expression (Fig. 2E). Therefore, rather than being discrete populations, these cells may exist as a differentiation continuum. Furthermore, a graded expression of monocyte genes, such as Ccr2, Lgals3 and Lsp1, was observed in MHC Ilhi BAMs, suggesting that these populations received monocyte input. When performing SCORPIUS trajectory inference ${ }^{29}$ on monocytes, MdCs and BAMs from the dura mater, these cells could be automatically ordered along a linear trajectory, moving from monocytes to MdCs, Di-BAMs and Dlo-BAMs (Fig. 2F). The most predictive genes were clustered into modules, which showed clear transcriptional gradients of genes that are lost or gained as cells would move from a monocyte state to mature BAMs (Fig. 2G).

We used the recently developed SCENIC pipeline ${ }^{30}$, which relies on co-expression and cis-regulatory motif analysis, to identify the gene regulatory networks and master regulators that drive the transcriptional state of microglia and BAMs. SCENIC analysis was performed on the pooled brain macrophage dataset and identified Gene Regulatory Networks, called "regulons". These regulons 
consist of a transcription factor and co-expressed genes containing the corresponding binding motif in its promoter region. Rather than measuring the activity of single genes, the SCENIC algorithm measures the activity of entire regulons, which is displayed in a binary activity matrix (Fig. 3A). tSNE plots were generated based on regulon activity and cells were colored by their matching Seurat clusters (Fig. 3B). This showed a clear separation between microglia and BAMs and more subtle distinctions between BAMs from different border regions. Potential master regulators for microglia included Sall1, Sall3, Mafb, Mef2a and IIf8, which have been previously implicated in regulating the microglial identity $9,14,15,31$, highlighting the accuracy of the SCENIC algorithm. SCENIC also identified master regulators that were absent/low in microglia but appeared active in all BAM subsets, including Spic, Ets2, Cebpb and members of the IRF and STAT family of transcription factors (Fig. 3A, C). Spic has been previously shown to control the development of splenic red pulp macrophages 32,33 and its regulon contained 212 predicted target genes (Supplementary Fig. 2). The Etv1 and Maf regulons were strongly enriched in MHC Illo meningeal BAMs (Fig. 3A, C). The Maf regulon contains 17 predicted genes, including Mrc1 and Lyve1 (Supplementary Fig. 2). Runx3, with its 77 predicted target genes (Supplementary Fig. 2), specifically marked MHC IIhi BAMs, while the $B c / 3$ regulon appeared active in all BAM subsets, except for SD-BAMs (Fig. 3A, C). As was observed previously (Supplementary Fig. 1), a fraction of CPlo-BAMs and CPhi-BAMs clustered separately from the main BAM subsets, solely due to the differential activity of regulons that drive IEGs. Interestingly, CPepi-BAMs, which clustered separate from BAMs and microglia, exhibited a transcriptional program that was driven by both microglia and BAM-specific transcription factors (TFs). Additional regulons/TFs that appeared active in these cells included Nfkb1, Maff, Relb, Atf3 and Nfe2l2 (Fig. 3A, C).

\section{BAM subsets can be identified at the protein level via high-dimensional fluorescence cytometry.}

To correlate single-cell gene with single-cell protein expression, we relied on high-dimensional fluorescence cytometry. Based on our scRNA-Seq data, we devised and optimized a myeloid-centered 19-color antibody panel (Fig. 4A). Flow cytometry was performed on the whole brain or on the individual brain borders. ISNE analysis was used to visualize the cytometry data in two dimensions and key markers that were differential between the various brain myeloid populations are shown in Fig. 4B. Microglia and BAMs could be identified based on a high expression of the macrophage markers $F 4 / 80$, CD64 and CX3CR1 and a low expression of CD11a, CD11c and Ly6C, which were associated with cDCs or monocytes. We devised an efficient manual gating strategy for selecting the different brain macrophage subsets in all samples (Fig. 4C and Supplementary Fig. 3A), which was mapped onto the tSNE plots (Fig. 4D). BAMs could be selected by gating for CD11alo $\mathrm{CX} 3 \mathrm{CR} 11^{\text {hiF }} 4 / 80^{\text {hi }}$ cells. Furthermore, in all brain regions two clearly outlined BAM subsets were observed, based on the expression of MHC II and MMR (CD206) (Fig. 4C). These subsets corresponded to the various BAM subsets that were identified by scRNA-Seq. Microglia were CD11 ${ }^{\text {lo }} \mathrm{CX} 3 \mathrm{CR} 1 \mathrm{hiF} 4 / 80^{\text {int }}$ and did not express MHC II or MMR. The CP also contained a $\mathrm{CD} 11 \mathrm{a}^{\mathrm{lo}} \mathrm{CX} 3 \mathrm{CR} 11^{\mathrm{h}} \mathrm{F} 4 / 80^{\mathrm{int}}$ fraction that was $\mathrm{MHC}$ I- MMR- and corresponded to CPepi-BAMs. In the dura, cells falling in the gate that identified microglia or CPepi-BAMs in other regions (Fig. 4C, orange gate) did not map to a specific population, confirming that a microglia-like 
subset is absent in this tissue. Other myeloid cells, including monocytes and CDCs, fell into the CD11 $a^{\text {hi }}$ fraction (Supplementary Fig. 3A). Therefore, using this 19-color panel, we could detect and gate all the previously identified BAM subsets with ratios that closely matched the ratios observed with our ScRNASeq approach. In addition, the various BAM subsets could also be identified and gated using more restricted 7-color panels (Supplementary Fig. 3B). Being able to identify the distinct BAM subsets via single-cell cytometry, not only confirmed our scRNA-seq data, but also allowed us to FACS sort them for complementary analyses and fate mapping studies.

The tissue-specific adaptation of BAMs from different border regions was also reflected at the level of their morphology. To visualize this, we optimized procedures for wholemount immunostaining of the dura and subdural meninges, which exposed the clearly distinct morphologies of subdural and dural BAMs (Fig. 4E-H). To ensure that the examined populations were resident macrophages, we relied on cell tracing in $C \times 3 c r 1$ CreER $\times$ R26-YFP double transgenic animals. Tamoxifen injections in these mice labels resident brain macrophages, which after a 4-week wash-out period are the only cell type in the CNS that remains YFP+34.

\section{Bulk RNA sequencing of BAM subsets further uncovers their transcriptional state and suggests} tissue-specific functional adaptations.

To confirm and complement our scRNA-Seq results and to further refine the transcriptional profile of BAMs, we FACS sorted BAMs for bulk RNA sequencing, which allows for a more in-depth profiling. To further assess inter-border variation, we opted to compare CPhi-BAMs with Dhi-BAMs (Fig. 5A). For intra-border heterogeneity, Dhi-BAMs were compared to Dlo-BAMs. Microglia were included as a reference population. In addition, to further unveil BAM-specific adaptations and gene signatures, we included tissue-resident macrophages from peripheral organs: peritoneal macrophages, lung alveolar macrophages and liver Kupffer cells. Principal component analysis of gene expression showed that brain macrophages clustered closer together, at a greater distance from peripheral macrophages (Fig. $5 \mathrm{~B})$. However, the various brain macrophage subsets also showed clear population-specific

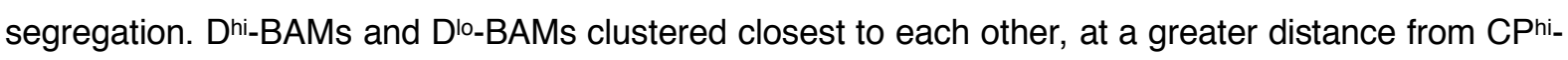
BAMs. Pearson correlation coefficients also showed the highest correlation between brain macrophages (Fig. 5C). Brain macrophages exhibited hundreds of differentially expressed genes when compared to all three peripheral macrophages (Fig. 5D). Microglia showed the highest number of uniquely upregulated genes (865), followed by CPhi-BAMs (275). Many significantly upregulated genes (706) were shared between all brain macrophage subsets. From the differentially expressed gene lists, we constructed heatmaps to reveal the signature gene expression of brain macrophages (Supplementary Fig. 4A, B).

When comparing CPhi-BAMs with Dhi-BAMs, we observed 1295 differentially expressed genes (Fig. $5 \mathrm{E})$. This confirms that BAMs from different border regions possessed unique transcriptional profiles.

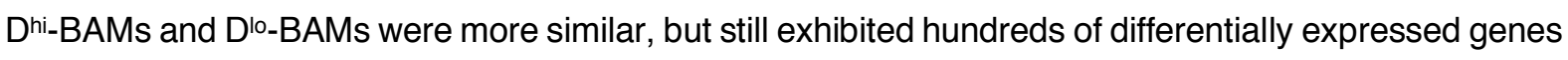
(Fig. 5F). 
To investigate how the transcriptional diversity of BAMs may relate to their putative functions, we examined the genes that were differential between BAMs versus microglia and used these to construct gene ontology $(\mathrm{GO})$ networks. GOs that were enriched in microglia related to known functions, including neurogenesis and TGF $\beta$ signaling (Supplementary Fig. 4C)8,13. Genes that were upregulated in all BAMs associated with GO categories linked to blood vessel development, lipid and cholesterol metabolism, and immune response and antigen presentation (Fig. 5G). Genes that were restricted to one or two BAM subsets revealed subset-specific GOs (Fig. 5H). CPhi-BAMs displayed enriched angiogenesis, ion transport and cell activation and migration. D-BAMs showed a general enrichment for GOs related to intracellular protein sorting and clathrin-mediated endocytosis, while the D'o-BAMs exhibited GOs associated with the negative regulation of immune response.

\section{Sall1+ CPepi-BAMs reside on the apical surface of the choroid plexus epithelium.}

Next, we further examined CPepi-BAMs, which exhibited an intriguing microglia-like phenotype. Since within BAMs only CPepi-BAMs expressed Sall1 (Fig. 2C), we used Sall1CreER x R26-YFP mice to trace these cells in the CP. Mice received tamoxifen injections and the CP was collected from the lateral or fourth ventricle for flow cytometric analysis. As shown previously ${ }^{14}$, Sall/1CreER efficiently labeled parenchymal microglia (Fig. 6B). Interestingly, we also observed that in the lateral and fourth CP $5.6 \%$ $\pm 0.9 \%$ and $2.6 \% \pm 0.5 \%$ of BAMs were YFP+, respectively (Fig. $6 \mathrm{~A}, \mathrm{~B}$ ). These numbers are in line with what we had observed via scRNA-Seq, where the CP-BAM B cluster (corresponding to CPepi-BAMs) was about $5 \%$ of the total BAM population (Fig. 1E). Sall1+ CP BAMs were MHC II-, CLEC12A- and expressed higher levels of CD11b as compared to Sall1- CP BAMs (Fig. 6C), mirroring what was seen at the gene level (Fig. 1E, 2D).

To investigate the spatial distribution of Sall1+ CP BAMs, we performed immunostaining on coronal brain sections of Sall1CreER $\times$ R26-YFP mice. YFP expression could be observed in a fraction of IBA1+ $\mathrm{CP}$ macrophages, confirming in situ that the CP contains a Sall1+ BAM subset (Fig. 6E). Interestingly, YFP+ IBA1+ BAMs were only observed on the apical surface of the CP epithelium, which was outlined via cytokeratin staining. Stromal IBA1+ macrophages were YFP-. In addition, while IBA1+ YFP- BAMs exhibited CLEC12A staining, this was not observed in YFP+ BAMs (Fig. 6F), in line with cytometry results (Fig. 6C). Lyz2 was another highly expressed BAM gene that was found to be low on the CPepiBAMs (Fig. 2D). Therefore, we examined the CP of $L y z 2^{\mathrm{GFP}}$ mice, where we observed that IBA1+ cells on the apical epithelial surface exhibited low Lyz2GFP expression as compared to their stromal IBA1+ counterparts (Fig. 6G). Together, these data show that Sall1+ BAMs, which correspond to the CPepiBAMs identified via scRNA-Seq, were epiplexus macrophages, likely to represent the elusive Kolmer's epiplexus cells ${ }^{20}$.

CPepi-BAMs from homeostatic brains share a core transcriptional signature with DiseaseAssociated Microglia that are observed under conditions of neurodegeneration.

As described earlier (Fig. 2C), CPepi-BAMs expressed microglial signature genes such as Tmem119, P2ry12, Selplg and Cx3cr1, but at lower levels compared to microglia (Fig. 7A). Conversely, an 
upregulation was seen of genes that are involved in lipid metabolism or phagocytosis, such as Cst7, Apoe, Clec7a, Lpl and Tryopb (Fig. 7A). Remarkably, this gene signature resembled that of the recently discovered Disease-Associated Microglia (DAM), which were first identified in the 5XFAD Alzheimer's disease $(A D)$ model ${ }^{18}$. To be able to compare CPepi-BAMs with DAM using the same single-cell platform, we performed 10x chromium scRNA-Seq analysis on CD45+ cells isolated from whole brains of APP/PS1 mice, a widely used AD model. Besides the immune cell populations that were observed in WT brains (Fig. 1B), APP/PS1 brains contained an additional microglial subset (Fig. 7B, C), which clearly exhibited the DAM gene signature that was described in 5XFAD mice (Fig. 7D). These data confirm the results of Keren-Shaul et al. and show that DAM from different AD models exhibit highly similar gene signatures. Interestingly, the differentially expressed genes seen in DAM (Fig. 7D) strongly resembled those of CPepi-BAMs (Fig. 7A). Comparing the top up- or downregulated genes of both subsets, showed that many were shared (Fig. 7E, F). This shows that the core DAM signature is not only observed under conditions of neurodegeneration or disease but is also induced in CPepi-BAMs in healthy young mice. However, there were also genes that were only enriched in one of the two populations. Examples include DAM-restricted upregulation of Trem2, Itgax, Ctsd, Baiap212, Cox6a2 and CPepi-BAM-restricted upregulation of Actr3b, or Folr2, Ttr, Mir155hg, Srxn1 and II1rn (Fig. 7E). A GO network, based on shared DAM and CPepi-BAM signature genes revealed GOs related to lipid metabolism, leukocyte differentiation and migration, and stimulus detection (Fig. 7F). Neuron development and regeneration, carbohydrate metabolism, and hypoxic response and blood vessel development were attributed to the DAM subset specifically. CPepi-BAM-specific GOs related to response to stimulus, cell migration, regulation of apoptosis and antigen processing and presentation (Fig. 7F).

It has been suggested that DAM are phagocytic cells ${ }^{16,18}$. We therefore investigated the phagocytic activity of CPepi-BAMs and compared it to that of parenchymal microglia. Brain or CP cells from Sall1CreER $x$ R26-YFP mice were incubated in vitro with pHrodo-labeled E. coli bioparticles, which become fluorescent only after entering the phagosome. Upon co-culture, we observed significantly higher pHrodo fluorescence in YFP+ CPepi-BAMs as compared to YFP+parenchymal microglia (Fig. 7G, $\mathrm{H})$, indicating that CPepi-BAMs exhibit a higher phagocytic activity.

\section{BAM subsets exhibit a mixed ontogeny and a postnatal development that is highly dependent on the border region.}

To investigate how BAM heterogeneity develops over time, we performed flow cytometry on dissected $\mathrm{CP}$, dura and subdural meninges starting from postnatal day (PD) 0 up to 50 weeks of age (Fig. 8A, B). This revealed that most BAMs shared a similar phenotype at birth, which was characterized by a high expression of MMR and absence of MHC II. The phenotype of SD-BAMs remained relatively stable, although a subtle decrease of MMR and a slight increase of MHC II expression was observed in older animals. However, the phenotype and composition of BAMs in the dura and CP dramatically changed from PD21 onwards (Fig. 8A). In the dura, BAMs downregulated MMR and an MMR ${ }^{\circ}$ MHC Il hi $^{\mathrm{hi}}$ population appeared, which became the predominant subset at 20W (Fig. 8A, B). A similar pattern 
occurred in the $\mathrm{CP}$, where BAMs more rapidly lost MMR expression, followed by the upregulation of MHC II. At 20W and onwards, more than $85 \%$ of CP BAMs were MHC Ilhi. with MHC Illo cells mostly representing CPepi-BAMs (Fig. 8A, B).

This shows that the BAM diversity seen at the different border regions in adult mice develops postnatally. This could be due to a phenotypical switch of resident BAMs and/or to a replacement by BM-derived macrophages. Therefore, we assessed the ontogeny of BAMs using various approaches. We relied on pulse-chase labeling in adult $C \times 3 c r 1$ CreER $\times$ R26-YFP mice to assess for bone marrow (BM)-input, by examining the dilution of YFP-labelled cells over time (Fig. 8C). As reported ${ }^{19}$, microglia labelling remained stable, indicating that these cells do not receive peripheral input. Similar observations were made for CPepi-BAMs and SD-BAMs, which did not display a significant reduction in YFP labeling. In contrast, YFP labelling gradually declined in dural and CP BAMs. This was most prominent in Dhi-BAMs, while a slower decline was observed in Dlo-BAMs and CPhi-BAMs. To confirm BM-derived replacement of BAMs, we used Flt3Cre $\times$ R26-YFP mice, in which Flt3-dependent definitive hematopoietic precursors are labelled, allowing for the distinction between BM- and embryonicallyderived macrophages ${ }^{35}$. YFP labelling was examined starting from PD0 up to $10 \mathrm{~W}$ of age (Fig. 8D). Classical blood monocytes were used as a reference population due to their exclusive BM origin. At birth, monocytes exhibited only a partial labeling, due to the low Flt3 promotor activity in embryos ${ }^{35}$, but this rapidly increased in the first postnatal week. Microglia and CPepi-BAMs displayed negligible YFP labelling at any of the examined time points. Absence of YFP expression in CPepi-BAMs was confirmed by immunohistochemistry (Fig. 8E). Only a minor fraction of SD-BAMs was YFP+ (1.4-9.7\%), which remained stable over the examined time period. CPlo-BAMs also did not display a large increase in YFP labelling, in accordance with the CX3cr1CreER pulse chase experiments (Fig. 8C), but this subset gradually disappeared. Other BAM subsets showed a progressive increase in YFP labeling. As soon as Di-BAMs were observed, the majority were YFP+ and at $10 \mathrm{~W}$ its YFP-labeling equaled that of monocytes. Replacement in Dlo-BAMs and CPhi-BAMs occurred more slowly. Together, these results indicate that microglia, CPepi-BAMs and SD-BAMs remained of embryonic origin, while other BAMs were gradually replaced by BM-derived precursors, although with very different kinetics depending on the subset and border region.

The signals that drive the heterogeneity of BAMs in the distinct border regions could be tissue-intrinsic or governed by peripheral stimuli. The latter may more strongly affect D-BAMs and CP-BAMs, since they reside in the proximity of fenestrated blood vessels at more permissive borders. Therefore, we assessed whether the microbiome affects the compositional shift of BAMs in these regions. The fraction of MHC IIhi BAMs was analyzed in the dura and CP of germ-free, SPF- and conventionally-housed mice at $10 \mathrm{~W}$ of age. This revealed that germ-free animals contained a significantly lower fraction of MHC IIhi BAMs in the $\mathrm{CP}$ and dura as compared to conventionally-housed mice (Fig. 8F). This suggests that the microbiome influences BAM heterogeneity. However, since the shift to MHC Il hi BAMs was still observed in germ-free animals, it also indicates that exposure to microorganisms is not strictly required. 


\section{Discussion}

Macrophages are an integral part of all organs and play an instrumental role in regulating tissue homeostasis ${ }^{8,36}$. While seminal studies have uncovered the transcriptional profile of microglia and their critical role in brain homeostasis and disease ${ }^{12,13,17,37}$, the phenotype and functions of other tissueresident brain macrophages have remained more elusive. We now provide extensive insights into the heterogeneity of BAMs and unveil their remarkable diversity, including the identification of a new microglia-like subset in the choroid plexus.

Recent work, which relied on mass cytometry of whole brain samples, has shown that the immune compartment of the brain is highly diverse, containing various lymphoid and myeloid subsets ${ }^{5-7}$. While mass cytometry offers valuable high-dimensional protein data, one limitation is the use of a limited and predefined panel of markers. Single-cell transcriptomics offers a genome-wide analysis and an unbiased characterization of cell types and activation states. Furthermore, analysis on dissected regions will reveal the regional diversity and identify minor populations that are easily missed when analyzing whole brain samples. For example, our scRNA-Seq analysis on dissected tissues showed that the composition of the brain DC pool varied in the distinct border regions and we identified brain DCs with a migratory gene signature that were not evident in previous studies 5 . All border regions contained a large number of macrophages or BAMs. The tissue environment has been shown to strongly impact the transcriptional profiles of resident macrophages ${ }^{9-11}$. Indeed, we observed that BAMs exhibited distinct transcriptional profiles as compared to microglia or peripheral macrophages. Genes that were upregulated in all BAMs included Apoe, Ms4a7, Mas4a6c and Cd36. This gene signature was predicted to be driven by SPIC and members of the STAT- and IRF family of transcription factors. Spic expression is regulated by heme and controls the development of splenic red pulp macrophages (RPM), which play an important role in iron homeostasis ${ }^{10,32,33}$. This may indicate some similarities between RPM and BAMs, which also lie in the proximity of blood vessels and may be exposed to heme. The expression of Apoe and other genes related to cholesterol and lipid metabolism, suggests a role for BAMs in lipid homeostasis. The brain constitutes a large lipid reservoir and has a specialized cholesterol metabolism with ApoE being the main cholesterol transporter in the $\mathrm{CNS}^{38}$. Besides a shared phenotype, BAMs also displayed clear border-specific signatures. Indeed, BAMs from the different border regions clustered separately from each other following scRNA-seq analysis. This was also confirmed at the bulk RNA-Seq level, where we observed hundreds of differentially expressed genes between $\mathrm{CP}$ and dural BAMs. This suggests that BAMs exhibit functional specialization tailored to the specific needs of the tissue environment. For example, CP-BAMs expressed Ttr, responsible for the shuttling of thyroid hormone from the blood into the $\mathrm{CSF}^{39}$ and Enpp2, responsible for the production of Lysophosphatidic acids (LPAs), important signaling molecules within the CNS ${ }^{40}$. Within each border region, BAMs could be further subdivided into two main subsets, which among others differed in the expression of class II major histocompatibility genes, Ccr2, Mrc1, Cd163, Cd38, Igf1 and Gas6. This corroborates a recent cytometry-based study that identified BAM subsets within whole brain samples based on the differential expression of MHC II, CCR2, CD38 and CD2065. Our scRNA-Seq analysis now offers a complete view of BAM heterogeneity, including an extensive list of signature genes for 
their identification. In addition, we reveal the relative abundance of BAM subsets at the various border regions and show how this heterogeneity developed over time, starting from birth. Subdural BAMs were mostly a homogenous population of MHC II- Lyve1 hi macrophages. In the CP and dura, MHC Ilhi BAMs only appeared after weaning age, but progressively increased and became the major fraction in adult mice. The microbiome, which has been shown to shape microglial development ${ }^{41}$, was partly involved in promoting the accumulation of MHC IIhi BAMs but was not the main determinant. MHC II expression in BAMs suggests a role in immune surveillance and antigen presentation. Macrophages in the meninges have been previously implicated in the antigen presentation to self-reactive T-cells during the induction of experimental autoimmune encephalomyelitis ${ }^{42}$.

ScRNA-Seq also revealed the presence of an additional BAM subset within the choroid plexus. Macrophage-like cells lining the apical side of the $\mathrm{CP}$ epithelium were previously discovered via electron microscopy and were called Kolmer's epiplexus cells, but their origins, phenotype and functions remained unknown ${ }^{20}$. We now show that CPepi-BAMs were macrophages with a transcriptional signature reminiscent of microglia and were found on the apical surface of the CP epithelium. These macrophages, which likely represent Kolmer's epiplexus cells, expressed highly specific microglial signature genes, including Sall1, which is even absent in BM-derived microglia that engraft the brain parenchyma under specific experimental conditions ${ }^{14,43}$. We show that, similar to parenchymal microglia ${ }^{19}$, epiplexus macrophages were strictly embryonically-derived and received no peripheral input. Therefore, we suggest that epiplexus macrophages are a subset of microglia that inhabit the CP epithelial niche. It is currently unknown whether microglial progenitors seed the CP during embryonic development and subsequently self-renew in situ, or whether throughout life there is a continuous influx of microglia from the subependymal parenchyma into the CP. The transcriptional profile of epiplexus macrophages was distinct from that of homeostatic parenchymal microglia but exhibited striking similarities to that of disease-associated microglia or DAM, which are observed under conditions of neurodegeneration ${ }^{16,18}$. Like epiplexus macrophages, DAM possess a clear microglial signature, but with decreased levels of "homeostatic" genes and an increase of phagocytosis and lipid metabolism genes $^{16,18}$. DAM are observed in a variety of neurodegenerative disease models ${ }^{16,18,44}$, which suggested that the DAM phenotype is a common microglial response to brain pathology ${ }^{44}$. Our results now extend this view and show that the core DAM signature is also observed in "homeostatic" epiplexus macrophages in healthy adults. We propose that the core DAM phenotype marks a second "accessory" function of microglia, with the first being neuronal support in the homeostatic parenchyma ${ }^{8}$. This second accessory function may relate to regulating tissue homeostasis, metabolism and responses to pathology. Which signals then drive the phenotype of epiplexus macrophages and what is their homeostatic function? The DAM signature has been shown to rely on TREM2/APOE/miR155dependent signaling16,18,45. Genes that in DAM were shown to be TREM2-dependent, were also upregulated in epiplexus macrophages, and we observed an upregulation of Apoe and Mir155hg, suggesting the involvement of the TREM2/APOE/miR155 pathway in epiplexus macrophages as well. Known TREM2 ligands are phospholipids and lipoproteins ${ }^{46-48}$. Epiplexus macrophages expressed many lipid metabolism genes (i.a. Apoe, Ldlr, Lpl, Ch25h, Fabp3). In addition, epiplexus macrophages 
exhibited upregulation of Hmox1, Srxn1 and Txnrd1, which are known to be induced by oxidized phospholipids via Nfe2l2-dependent signaling ${ }^{49}$, a regulon that was also enriched. We speculate that the phenotype of epiplexus macrophages, which are in direct contact with the CSF and its lipoprotein content, is shaped through TREM2-based lipid-sensing and that these cells play a role in the brain's lipid metabolism. How epiplexus macrophages regulate lipid homeostasis and whether its dysregulation can affect the development of disease remain interesting open questions.

During embryonic development tissue-resident macrophages may derive from yolk sac macrophages, fetal liver monocytes or hematopoietic stem cells (HSCs) ${ }^{36}$. Upon birth, some organs show a progressive replacement of embryonic macrophages by BM-derived cells, while other macrophage populations such as microglia remain of embryonic origin ${ }^{36}$. Recent work has shown that subdural and perivascular BAMs also have an embryonic origin and self-maintain independent of the $\mathrm{BM}$, while $\mathrm{CP}$ BAMs have a mixed ontogeny ${ }^{19}$. Our results now confirm and extend these observations. We show that all dural and stromal CP BAM subsets were gradually replaced by BM-derived progenitors, but that this happened with distinct kinetics. Monocytes preferentially gave rise to MHC Ilhi BAMs, while MHC IIo BAMs in the dura and stromal CP were gradually lost. At 10 weeks, Dhi-BAMs were completely BMderived, while replacement of CPhi-BAMs was markedly slower. Trajectory inference also showed a clear differentiation continuum between monocytes and BAMs in the dura, highlighting the power of scRNA-Seq for ontogeny studies. In contrast, epiplexus and subdural BAMs were not replaced by monocytes. This indicates that at steady-state, the macrophage pool in the brain's more permeable regions (dura mater and CP stroma) showed BM-replacement, while this did not occur in the brain's closed tissues (parenchyma, subdural meninges and the apical surface of the CP epithelium). This suggests that tissue accessibility is a major determinant for brain macrophage ontogeny, which may support the concept of niche competition ${ }^{50}$. Furthermore, it indicates that besides the tissue environment, also ontogeny shapes some of the transcriptional diversity that is observed in brain macrophages. The highly distinct transcriptional profile of epiplexus vs. stromal CP BAMs is likely to be driven in part by their distinct ontogeny. Many transcripts in MHC Ilhi BAMs were also a remnant of their monocytic past, which were gradually being lost. However, while CPhi-BAMs or Dlo-BAMs had a mixed ontogeny, we did not observe two distinct CPhi-BAM or Dlo-BAM subsets at the single-cell transcriptome level, suggesting that for these populations embryonic and monocyte-derived BAMs were equivalent. In conclusion, our findings help to unravel the complexity of the brain's macrophage compartment. We provide insights into the heterogeneity, functionality and ontogeny of brain macrophages. These results offer a framework that will enable future studies aiming to further unveil the involvement of brain macrophages in health and disease. 


\section{Acknowledgements}

This works was supported by Innoviris (Attract grant) and Research Foundation Flanders (FWO) to K. Movahedi. H. Van Hove is supported by an FWO predoctoral fellowship. We thank Yvon Elkrim for technical assistance, Mate Kiss for helpful discussions and VIB Tech Watch for support and funding with regard to single-cell RNA sequencing technologies. 


\section{References}

1. Shechter, R., London, A. \& Schwartz, M. Orchestrated leukocyte recruitment to immuneprivileged sites: absolute barriers versus educational gates. Nat Rev Immunol 13, 206-218 (2013).

2. Engelhardt, B., Vajkoczy, P. \& Weller, R. O. The movers and shapers in immune privilege of the CNS. Nat. Immunol. 18, 123-131 (2017).

3. Louveau, A. et al. Structural and functional features of central nervous system lymphatic vessels. Nature 523, 337-341 (2015).

4. Wolburg, H. \& Paulus, W. Choroid plexus: Biology and pathology. Acta Neuropathol. 119, 7588 (2010).

5. Mrdjen, D. et al. High-Dimensional Single-Cell Mapping of Central Nervous System Immune Cells Reveals Distinct Myeloid Subsets in Health, Aging, and Disease. Immunity 48, 599 (2018).

6. Ajami, B. et al. Single-cell mass cytometry reveals distinct populations of brain myeloid cells in mouse neuroinflammation and neurodegeneration models. Nat. Neurosci. 21, 541-551 (2018).

7. Korin, B. et al. High-dimensional, single-cell characterization of the brain's immune compartment. Nat. Neurosci. 20, 1300-1309 (2017).

8. Okabe, Y. \& Medzhitov, R. Tissue biology perspective on macrophages. Nat. Immunol. 17, 917 (2016).

9. Lavin, Y. et al. Tissue-resident macrophage enhancer landscapes are shaped by the local microenvironment. Cell 159, 1312-1326 (2014).

10. Gautier, E. L. et al. Gene-expression profiles and transcriptional regulatory pathways that underlie the identity and diversity of mouse tissue macrophages. Nat. Immunol. 13, 11181128 (2012).

11. Gosselin, D. et al. Environment drives selection and function of enhancers controlling tissuespecific macrophage identities. Cell 159, 1327-1340 (2014).

12. Prinz, M. \& Priller, J. Microglia and brain macrophages in the molecular age: from origin to neuropsychiatric disease. Nat. Rev. Neurosci. 15, 300-312 (2014).

13. Butovsky, O. et al. Identification of a unique TGF- $\beta$-dependent molecular and functional signature in microglia. Nat. Neurosci. 17, 131-143 (2013).

14. Buttgereit, A. et al. Sall1 is a transcriptional regulator defining microglia identity and function. Nat. Immunol. 17, 1397-1406 (2016).

15. Matcovitch-Natan, O. et al. Microglia development follows a stepwise program to regulate brain homeostasis. Science 353, aad8670-1-12 (2016).

16. Krasemann, S. et al. The TREM2-APOE Pathway Drives the Transcriptional Phenotype of Dysfunctional Microglia in Neurodegenerative Diseases. Immunity 47, 566-581 (2017).

17. Hickman, S. E. et al. The microglial sensome revealed by direct RNA sequencing. Nat. Neurosci. 16, 1896-1905 (2013).

18. Keren-Shaul, H. et al. A Unique Microglia Type Associated with Restricting Development of Alzheimer's Disease. Cell 169, 1276-1290 (2017).

19. Goldmann, T. et al. Origin, fate and dynamics of macrophages at central nervous system interfaces. Nat. Immunol. 17, 797-805 (2016).

20. Ling, E.-A., Kaur, C. \& Jia, L. Origin, Nature, and Some Functional Considerationsof Intraventricular Macrophages, With Special Referenceto the Epiplexus Cells. Microsc. Res. Tech. 41, 43-56 (1998).

21. Lun, A. T. L., McCarthy, D. J. \& Marioni, J. C. A step-by-step workflow for low-level analysis of single-cell RNA-seq data with Bioconductor. F1000Research 5, 2122 (2016).

22. Satija, R., Farrell, J. A., Gennert, D., Schier, A. F. \& Regev, A. Spatial reconstruction of singlecell gene expression data. Nat. Biotechnol. 33, 495-502 (2015).

23. Miller, J. C. et al. Deciphering the transcriptional network of the dendritic cell lineage. Nat. Immunol. 13, 888-899 (2012).

24. Guilliams, M. et al. Unsupervised High-Dimensional Analysis Aligns Dendritic Cells across Tissues and Species. Immunity 45, 669-684 (2016).

25. Mildner, A. et al. Genomic Characterization of Murine Monocytes Reveals C/EBP $\beta$ Transcription Factor Dependence of Ly6C- Cells. Immunity 46, 849-862 (2017).

26. Fourgeaud, L. et al. TAM receptors regulate multiple features of microglial physiology. Nature 532, 240-244 (2016). 
27. van den Brink, S. C. et al. Single-cell sequencing reveals dissociation-induced gene expression in tissue subpopulations. Nat. Methods 14, 935-936 (2017).

28. Haimon, Z. et al. Re-evaluating microglia expression profiles using RiboTag and cell isolation strategies. Nat. Immunol. 19, 636-644 (2018).

29. Cannoodt, R., Saelens, W., Sichien, D. \& Tavernier, S. SCORPIUS improves trajectory inference and identifies novel modules in dendritic cell development. bioRxiv 079509 (2016).

30. Aibar, S. et al. SCENIC: Single-cell regulatory network inference and clustering. Nat. Methods 14, 1083-1086 (2017).

31. Kierdorf, K. et al. Microglia emerge from erythromyeloid precursors via Pu.1- and Irf8dependent pathways. Nat. Neurosci. 16, 273-280 (2013).

32. Kohyama, M. et al. Role for Spi-C in the development of red pulp macrophages and splenic iron homeostasis. Nature 457, 318-321 (2009).

33. Haldar, M. et al. Heme-mediated SPI-C induction promotes monocyte differentiation into ironrecycling macrophages. Cell 156, 1223-1234 (2014).

34. Goldmann, T. et al. A new type of microglia gene targeting shows TAK1 to be pivotal in CNS autoimmune inflammation. Nat. Neurosci. 16, 1618-1626 (2013).

35. Gomez Perdiguero, E. et al. Tissue-resident macrophages originate from yolk-sac-derived erythro-myeloid progenitors. Nature 518, 547-551 (2014).

36. Ginhoux, F. \& Guilliams, M. Tissue-Resident Macrophage Ontogeny and Homeostasis. Immunity 44, 439-449 (2016).

37. Gosselin, D. et al. An environment-dependent transcriptional network specifies human microglia identity. Science 356, 1248-1259 (2017).

38. Leduc, V., Jasmin-Bélanger, S. \& Poirier, J. APOE and cholesterol homeostasis in Alzheimer's disease. Trends Mol. Med. 16, 469-477 (2010).

39. Richardson, S. J., Wijayagunaratne, R. C., D'Souza, D. G., Darras, V. M. \& Van Herck, S. L. J. Transport of thyroid hormones via the choroid plexus into the brain: The roles of transthyretin and thyroid hormone transmembrane transporters. Front. Neurosci. 9, 1-8 (2015).

40. Yung, Y. C., Stoddard, N. C., Mirendil, H. \& Chun, J. Lysophosphatidic Acid Signaling in the Nervous System. Neuron 85, 669-682 (2015).

41. Thion, M. S. et al. Microbiome Influences Prenatal and Adult Microglia in a Sex-Specific Manner. Cell 172, 500-516 (2018).

42. Kivisakk, P. et al. Localizing central nervous system immune surveillance: Meningeal antigenpresenting cells activate T cells during experimental autoimmune encephalomyelitis. Ann. Neurol. 65, 457-469 (2009).

43. Cronk, J. C. et al. Peripherally derived macrophages can engraft the brain independent of irradiation and maintain an identity distinct from microglia. J. Exp. Med. 215, 1627-1647 (2018).

44. Deczkowska, A. et al. Disease-Associated Microglia: A Universal Immune Sensor of Neurodegeneration. Cell 173, 1073-1081 (2018).

45. Butovsky, O. et al. Targeting miR-155 restores abnormal microglia and attenuates disease in SOD1 mice. Ann. Neurol. 77, 75-99 (2015).

46. Song, W. et al. Alzheimer's disease-associated TREM2 variants exhibit either decreased or increased ligand-dependent activation. Alzheimer's Dement. 13, 381-387 (2017).

47. Wang, Y. et al. TREM2 Lipid Sensing Sustains the Microglial Response in an Alzheimer's Disease Model. Cell 160, 1061-1071 (2015).

48. Yeh, F. L., Wang, Y., Tom, I., Gonzalez, L. C. \& Sheng, M. TREM2 Binds to Apolipoproteins, Including APOE and CLU/APOJ, and Thereby Facilitates Uptake of Amyloid-Beta by Microglia. Neuron 91, 328-340 (2016).

49. Kadl, A. et al. Identification of a Novel Macrophage Phenotype That Develops in Response to Atherogenic Phospholipids via Nrf2. Circ. Res. 107, 737-746 (2010).

50. Guilliams, M. \& Scott, C. L. Does niche competition determine the origin of tissue-resident macrophages? Nat. Rev. Immunol. 17, 451-460 (2017). 


\section{Figure Legends}

Figure 1. Single cell RNA-sequencing of discrete brain compartments reveals regional immune cell heterogeneity. (a) Schematic overview of the 10x chromium single-cell RNA sequencing procedure on CD45+ immune cells isolated from the whole brain and micro-dissected border regions of 9-week-old C57BL/6J mice. (b) tSNE plot of 6530 cells isolated from the whole brain, showing the immune cell populations that were identified via unsupervised clustering. (c) tSNE plot of 6564 cells isolated from the enriched subdural meninges (SDM). (d) tSNE plot of 5865 cells isolated from the dura mater. (e) tSNE plot of 3940 cells from the choroid plexus (following regress out of IEGs, see Supplementary Fig. 1). (c-e) Dot plots corresponding to the tSNE plots of the border regions display the expression of subset-specific genes, with the size of the dot representing the percentage of cells expressing the gene and the color representing its average expression within a cluster. The $\%$ of each cluster within the total population is mentioned on the right side of each dot plot. (f) Heatmap of normalized expression for selected genes in DC subsets and other indicated populations in the Dura Mater. (g) Pie charts showing the proportion of the different DC subsets within each border region (SubDural meninges, Dura and CP). *Sub-clusters within the macrophage populations were omitted for clarity. ${ }^{* *}$ Cluster was manually selected. cl. Monocytes: classical monocytes, non-cl. Monocytes: nonclassical monocytes, MdC: monocyte-derived cells, MC: monocytic cells.

Figure 2. Border-associated macrophages are heterogeneous and exhibit tissue-specific transcriptional signatures. (a) tSNE plot of 10.947 brain macrophages pooled from the whole brain and border regions revealing the existence of 6 BAM subsets. (b) Similar to (a) but with the colors representing the tissue origin of cells. Magenta: whole brain; red: CP; blue: Dura; green: SDM, (c) Bar plots showing the mean log2 normalized counts of previously reported microglial signature genes in microglia and the BAM subsets that were identified in (a). Gene expression levels in classical monocytes from the dura mater (Mo) are included as an additional reference. (d) Heatmap of normalized gene expression in microglia and the BAM subsets that were identified in (a). Genes are grouped based on their signature expression in the indicated BAM subsets. (e) tSNE maps showing the expression of various BAM signature genes. The color code (gold: low expression, red: high expression) and the range of the log2 normalized counts are shown on the lower right of each plot. (f) SCORPIUS trajectory inference on classical monocytes, MdCs and BAMs from the dura mater. Cells were automatically ordered along a linear trajectory as shown. (g) The top 100 genes were clustered into 7 gene modules (normalized expression) that correspond to the transition from monocyte to BAMs.

Figure 3. SCENIC reveals the gene regulatory networks and master regulators that drive the transcriptional program of BAMs. (a) SCENIC was performed on 10.947 brain macrophages pooled from the whole brain and border regions (see $2 a$ ), yielding a binary activity matrix that predicts whether the indicated regulons are active in individual cells. Cells were clustered based on their regulon activity. The colors of the individual cells (shown at the top of the activity matrix) match the color of the clusters that were identified based on gene expression using seurat (see $3 b$ top). Important regulons are shown 
for the various macrophage subsets: Microglia (orange), general BAMs (green), MHC Il० BAMs (blue), MHC Ilhi BAMs (red), CPepi-BAM (pink) (b) tSNE plot based on gene expression (top) and tSNE plot based on the binary regulon activity matrix (bottom). Cells are colored based on their designation to the clusters in the expression-based tSNE. (c) tSNE plots showing the activity of the indicated regulons, together with the enriched motif for that TF. Cells in which the indicated regulon is active (i.e. regulon activity exceeds a regulon-specific threshold) are marked in blue.

Figure 4. Identification of BAM subsets using high-dimensional fluorescence cytometry. (a) Single cells from the whole brain and border tissues of 9-week-old C57BL/6J mice were stained with a 19-color antibody panel for fluorescence cytometry on the FACSymphony. (b) viSNE plots from the whole brain, enriched SDM, dura and CP showing expression of the indicated markers. (c) Depiction of the manual gating strategy used for identifying microglia and BAM subsets in the whole brain or dissected border regions. Microglia and CPepi-BAMs were gated as CD11b+CD11aloCX3CR1hiF4/80intMMR-MHC II- cells. For other BAM subsets, we first selected $C D 11 b+C D 11 a^{\text {lo }} C X 3 C R 1^{\text {hiF }} 4 / 80^{\text {hi }}$ cells, followed by gating of MMR hHC $\|^{\text {lo }}$ and MMRint MHC $\|^{\text {hi }}$ BAMs.

(d) Manually gated myeloid populations (see also supplementary Fig. 3) were mapped onto the viSNE plot of the whole brain, enriched SDM, dura and choroid plexus. cl. Monocytes: classical monocytes, non-cl. Monocytes: non-classical monocytes. (e) Legend corresponding to (f-h) depicting the in situlocation of the different BAM subsets. (f) Wholemount of pia mater, (g) wholemount of dura mater and (h) coronal section of the LV CP from tamoxifen-treated Cx3cr1CreER $x$ R26-YFP mice, following a 4week washout period. SD-BAMs were identified as LYVE1+YFP+ cells in (f), while Dural and CP BAMs were identified as IBA1+YFP+ cells in $(g, h)$ respectively. Dural blood vessels and choroid plexus epithelium were stained with anti-CD31 and anti-Cytokeratin, respectively. Scale bars: $100 \mu \mathrm{m}$.

Figure 5. Bulk RNA-sequencing of BAM subsets suggests tissue-specific functional adaptations. (a) Shematic overview of bulk RNA-seq on various brain and peripheral macrophages. PMF: peritoneal macrophages, AMF: alveolar macrophages, KC: kupffer cells. (b) Principal component analysis of the $15 \%$ most variable genes for brain and peripheral macrophage populations. (c) Pearson correlation matrix of brain and peripheral macrophages based on gene expression. (d) Genes that were significantly higher in microglia or BAM subsets as compared to all three peripheral macrophages (PMFs, AMFs, KCs) are shown in the Venn diagram. (Adj-p $<0.01, \log 2 F C>1$ ) (e-f) Volcano plot displaying genes that are DE (Adj-p $<0.01,|\log 2 F C|>1$ ) between (e) CPhi-BAMs vs. Dhi-BAMS and (f) Di-BAMs vs. Dlo-BAMs. Blue dots represent DE genes with mean normalized counts $<30$, red dots represent DE genes with mean normalized counts > 30. (g-h) Gene Ontology (GO) Network based on genes that are DE between BAM subsets vs. microglia. Venn diagram displays unique and intersecting DE genes among the different BAM subsets. Each node represents a gene ontology. A selection of genes associated to each cluster of GOs is displayed. (g) GO Network based on DE genes shared by all BAM subsets with a log2FC >1. (h) GO Network based on DE genes unique to CPhi-BAMs (yellow), 
Dhi-BAMs (red), Dlo-BAMS (orange), shared by D-BAMs (green), shared by CPhi- and Dhi-BAMs (blue) with a $\log 2 \mathrm{FC}>1$.

Figure 6. Sall1+ CPepi BAMs reside on the apical surface of the choroid plexus epithelium. Sall1+ CP-BAMs were identified using Sall1CreER $x$ R26-YFP reporter mice. (a) Gating strategy used for identifying Sall1+ CP-BAMs. (b) Percentage of YFP+ cells within microglia, lateral ventricle CP-BAMs and 4 th ventricle CP-BAMs. Bars represent mean \pm SEM of $n=15 \mathrm{M} / \mathrm{F}$ mice (microglia) and $5 \mathrm{M} / \mathrm{F}$ mice (CP). (c) Comparison of CD11b vs. MHC II, CD11b vs. CD45, and CLEC12A expression between YFP+ and YFP- CP-BAMs. (d) Legend corresponding to (e-g) depicting the in situ-location of the different BAM subsets. (e-f) Three-color IHC performed on coronal cryo-sections of brains from tamoxifeninjected Sall1CreER $\times$ R26-YFP mice. (e) Sections containing the lateral, third and fourth ventricle CP were stained with anti-IBA1 (red), anti-Cytokeratin (blue) and anti-GFP/YFP (green). Upper panels show all three channels, lower panels show green and blue channel only. (f) Sections containing the lateral ventricle CP were stained with anti-IBA1 (red), anti-CLEC12A (blue) and anti-GFP/YFP (green). Left panel shows all three channels, right panel shows green and blue channel only. (g) Coronal cryosections of adult $L y z 2-$ GFP reporter mice were stained with anti-IBA1 (red), anti-Cytokeratin (blue) and anti-GFP/YFP (green). full arrows: IBA1+YFP+ (d-e) or GFP- (f) BAMs on the apical side of the choroid plexus epithelium (pink in d); dashed arrows: IBA1+ YFP- (d-e) or GFP+ (f) BAMs within the choroid plexus stroma (blue in d). Scale bars: $50 \mu \mathrm{m}$ (e and g), $25 \mu \mathrm{m}$ (f).

Figure 7. CPepi-BAMs share a common transcriptional profile with Disease-Associated Microglia (DAM) found in Alzheimer's disease models. (a) Volcano plot showing genes that are DE (-log10 Adj-p $>20$, I log2FC I >1) between CPepi-BAM and microglia in red. (b) tSNE plot of 3418 CD45+ cells from the whole brain of 9M old male APP/PS1 mice showing the presence of a DAM cluster that was identified via unsupervised clustering. (c) DAM in APP/PS1 mice express Cst7 and low levels of homeostatic microglial genes such as Tmem119. (d) Volcano plot showing genes that are DE (-log10 Adj-p > 20, I log2FC I >1) between DAM and microglia in red. (e) Scatter plot comparing the log10 Adj$p$ value of the 100 most significantly upregulated genes in CPepi-BAMs vs. microglia ( $x$-axis) and in DAM vs. microglia (y-axis). The sign of the log10 Adj-p corresponds to up or downregulation of genes. Common up and downregulated genes are found within the red and blue squares, respectively. (f) GO Network based on the genes that are DE $(-\log 10$ Adj $p>10$ and $\log 2 F c>1)$ between CPepi-BAMs vs. microglia or DAM vs. microglia (unique and shared genes are displayed in a Venn diagram). The GOs shared by both subsets (purple) or unique to CPepi-BAMs (dark blue) or DAM (orange) are shown. A selection of genes associated to each cluster of GOs is displayed. (g-h). Comparison of the phagocytic capacity of CPepi-BAMs and microglia in tamoxifen-treated Sall1CreER $x$ R26-YFP mice using pHrodolabeled E. coli bioparticles. (g) CP and brain single-cell suspensions were incubated with pHrodolabeled $\mathrm{E}$. coli bioparticles at $0^{\circ} \mathrm{C}$ (brain) or at $37^{\circ} \mathrm{C}$ (brain and $\mathrm{CP}$ ). Histograms show pHrodo fluorescence in YFP+ microglia and YFP+CPepi-BAMs. (h) pHrodo Median Fluorescence Intensity (MFI) (left) and \% of pHrodo ${ }^{+}$cells (right) in microglia and CPepi-BAMs. Bars represent mean \pm SEM of $n=3$ 
with 2 male adult mice pooled/ repeat. Significance was evaluated using an unpaired two-tailed t-test. ${ }^{* *} p$-value $<0.01,{ }^{* * *} p$-value $<0.001,{ }^{* * * *} p$-value $<0.0001$. MFI p-values (Microglia $37^{\circ} \mathrm{C}$ vs. Microglia $0^{\circ} \mathrm{C}:<0.0001$, CPepi-BAM vs. Microglia $\left.37^{\circ} \mathrm{C}: 0.0019\right) \%$ pHrodo ${ }^{+}$cells $\mathrm{p}$-values (Microglia $37^{\circ} \mathrm{C}$ vs. Microglia $0^{\circ} \mathrm{C}:<0.0001$, CPepi-BAM vs. Microglia $\left.37^{\circ} \mathrm{C}: 0.0003\right)$.

Figure 8. BAM subsets exhibit a mixed ontogeny and display a region-specific postnatal

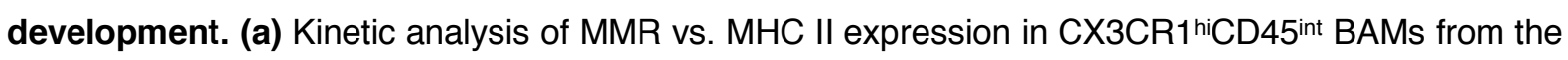
enriched SDM, Dura and Choroid plexus starting from PDO up to $50 \mathrm{~W}$ of age. (b) $\%$ of MHC Illi cells within the BAM populations from PD0-50W. Bars represent the mean \pm SEM of $n=4$ animals per time point. (c) Analysis of YFP dilution over time in adult Cx3cr1CreER $\times$ R26-YFP mice that received tamoxifen injections at 3-4 weeks of age. Populations were examined from $4 \mathrm{~W}$ to $33 \mathrm{~W}$ post-tamoxifen injections via flow cytometry. Each time point represents the mean \pm SEM of $n=4$ animals, except $n=3$ at 20W. The decrease in YFP labelling was evaluated with ONE-WAY ANOVA. Microglia ( $p$-value: 0.076, df: 4, F: 2.7), CPepi-BAM (p-value: 0.269, df: 4, F: 1.4), CPlo-BAM (p-value: 0.004,df: 4, F: 6,2), CPhi-BAM ( $p$ value <0.001, df: 4, F: 31,6), Dlo-BAM ( $p$-value: <0.001, df: 4, F: 10.0), Dhi-BAM ( $p$-value: $<0.001$, df: 4, F: 63.1), SD-BAM (p-value: 0.076, df:3, F: 3.0) (d) Evaluation of BM contribution to BAMs and microglia from PD0 to $10 \mathrm{~W}$ using Flt3Cre $\times$ R26-YFP mice. The $\%$ of YFP+ cells was evaluated with flow cytometry in blood monocytes (CD45+CD11b+Ly6chiLy6G) (purple) and the indicated brain macrophage populations. The colors correspond to those of the populations in panel A. Bars represent mean $\pm S E M$ of $n=6$ (PD0), $n=6$ (PD3), $n=4$ (PD6), $n=5$ (3W), $n=4(6 W), n=4(10 W)$. The increase in YFP labelling was assessed with ONE-WAY ANOVA between $3 \mathrm{~W}$ and 10W. Blood MO ( $p$-value: 0.1, df:2, F: 2.4) CPepi-BAM (0.4, df: 1, F: 1) Microglia (p-value: 0.8, df: 2, F: 0.2), SD-BAM (p-value: 0.1, df:2, F: 3.0), CPlo-BAM (p-value: 1, df: 2, F: 0.01), CPhi-BAM (p-value: 0.01, df: 2, F: 7.1), Dlo-BAM (p-value < 0.001, df: 2, F: 26.1), Dhi-BAM (p-value <0.001, df: 2, F: 28.6). (e) IHC on coronal cryo-sections of 6Wold Flt3 ${ }^{\text {re }} \times$ R26-YFP mouse containing the LV CP. Sections were stained with anti-IBA1 (red), antiCytokeratin (blue) and anti-GFP/YFP (green). Dashed arrow: YFP+ stromal CP-BAM, full arrow: YFPCPepi-BAM. scale bar: $50 \mu \mathrm{m}$. (f) Percentage of MHC II hi CP-BAMs and D-BAMs in 10W-old Germ-free (GF), SPF and conventional animal facility (conv) C57BL/6 mice. Bars represent mean \pm SEM of $n=4$ $\mathrm{M} / \mathrm{F}$ mice (GF CP), $\mathrm{n}=8 \mathrm{M} / \mathrm{F}$ mice (GF Dura), $\mathrm{n}=10 \mathrm{M}$ mice (conventional and SPF). The significance was evaluated using an unpaired two-tailed t-test. ns $p$-value $>0.05$, ${ }^{*} p$-value $<0.05$, ${ }^{* *} p$-value $<0.01$, ${ }^{* \star *} p$-value < 0.001 . CP p-values (SPF vs. conv: 0.02 , GF vs. SPF: 0.25 , GF vs. conv: 0.0006 ). D pvalues (SPF vs. conv: 0.11 , GF vs. SPF: 0.007 , GF vs. conv: 0.0002 ). 


\section{Legends of supplementary figures}

Supplementary Figure 1: Heterogeneity in choroid plexus BAMs at the level of immediate early genes. (a) tSNE plot of 3940 cells from the choroid plexus. This shows two main macrophage clusters: CP-BAM A1 and CP-BAM A2, in addition to the smaller macrophage cluster CP-BAM B. (b) Expression of prototypical macrophages genes such as Csf1r, Adgre1 and Fcgr1 confirms that CP-BAM A1, CPBAM A2 and CP-BAM B are macrophages. (c) Volcano plot showing the genes that are DE between CP-BAM A1 vs. CP-BAM A2 in red. (d) tSNE plot following regress out of IEGs. This shows that CP BAM-A1 and CP-BAM A2 now cluster as a single population. (e) tSNE maps showing the expression of H2-Aa, Mrc1, Gas6 and Ccr2 before and after IEG regress out. BAM heterogeneity at the level of these genes is more evident after regressing out EGs, showing that IEG induction can overshadow potentially more interesting biological heterogeneity.

Supplementary Figure 2. Selected regulons from SCENIC. SCENIC regulons with the regulator (green) and a selection of the regulated genes (white circle) visualized with iRegulon in cytoscape. The regulons of the indicated TFs are shown, together with the corresponding motif and the number of genes within the regulon.

Supplementary Figure 3: Gating strategy for the flow cytometric analysis of brain myeloid cells.

(a) Gating strategy for dendritic cells ( $\mathrm{CDC} 1$ and $\mathrm{CDC} 2$ ), neutrophils, monocytes (classical and nonclassical) and MdCs shown for whole brain samples. (b) Gating strategy for BAMs and microglia based on 7 markers. All brain macrophages were CD45+ and CD11 bi hi. Doublets and dead cells were excluded. All BAMs were gated as $C X 3 C R 1^{\text {hi }} C D 45^{\text {int }}$ cells and microglia were gated as $C X 3 C R 1^{\text {hi }} C D 45^{\text {lo }}$ cells. CP-BAMs were further subdivided in MHC II'oCLEC12A $1{ }^{10}$ CPepi-BAMs (green) and CLEC12A+ CPloBAMs or CPhi-BAMs (orange). D-BAMs were further subdivided into Dlo-BAMs (MMR ${ }^{\text {hi }}$ MHC Illo, green)

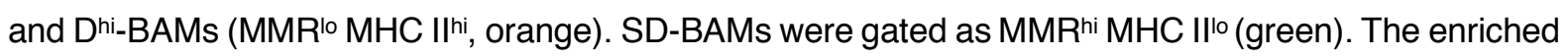
SDM also contained MMR-MHC II- microglia (orange). The different BAM gates were overlaid on the tSNE plots of single cells. Brains and border regions were dissected from 20W old C57BL/6 mice.

\section{Supplementary Figure 4. Transcriptional signatures of brain macrophages as revealed by bulk}

RNA sequencing. (a) Gene expression heatmap showing genes that are high in brain macrophages and low in the examined peripheral macrophages. Genes are grouped into cell surface (yellow), intracellular (blue), secreted protein (SP) (green) and transcription factor (TF) (magenta) genes. (b) Heatmap showing microglial signature genes. The genes are ordered based on the expression levels in CPhi-BAMs. (c) Gene Ontology (GO) Network based on genes that are DE between BAM subsets and microglia. The Network is based on DE genes shared by all BAMs and enriched in microglia with a $\log 2 \mathrm{FC}<-1$. 
A

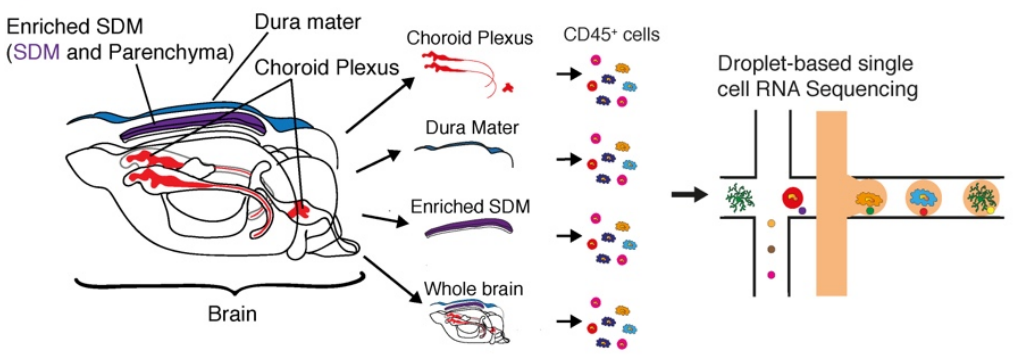

B Whole brain Neû́trophils

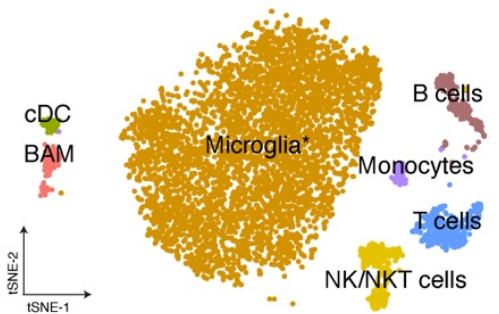

E Choroid plexus
C Enriched subdural meninges

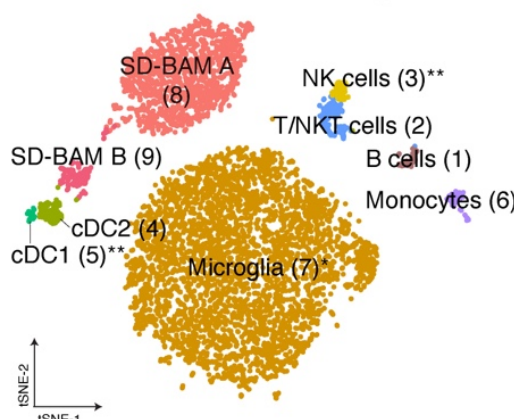

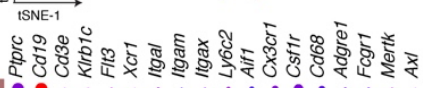

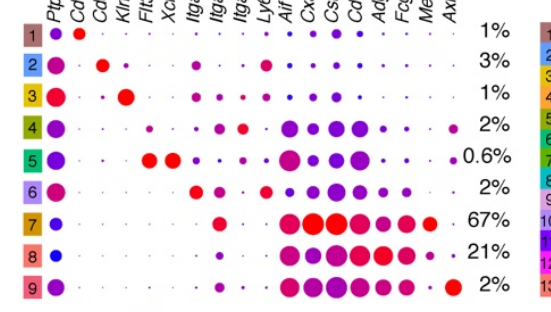

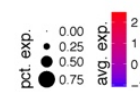

F

$$
\begin{array}{r}
\text { CDC2 } \\
\text { CDC1 } \\
\text { pDC } \\
\text { migDC } \\
\mathrm{D}^{\text {IO }} \text {-BAM } \\
\mathrm{D}^{\text {hi-BAM }} \\
\text { MdC } \\
\text { C. Mono } \\
\text { N.C. Mono } \\
\text { Figure } 1 .
\end{array}
$$

Figure 1.
D Dura mater

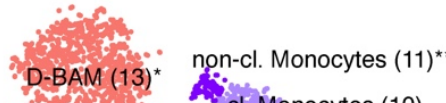

P-BAM (13) ${ }^{\star}$ Al. Monocytes (10) - Mad (12) MC (9)

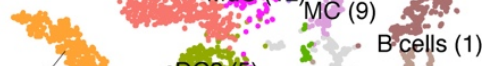

(4) Neutrophils $\mathrm{CDC}_{2}(5)$

$$
\text { कDCf7) TANKT cells (2) }
$$$$
\operatorname{migDC}(8) \cdots \text { (3) NK cells }
$$$$
\underset{\text { ISNE-1 }}{\uparrow} \quad \mathrm{CDCl}^{-}
$$

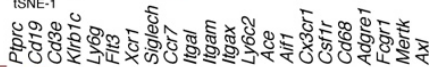

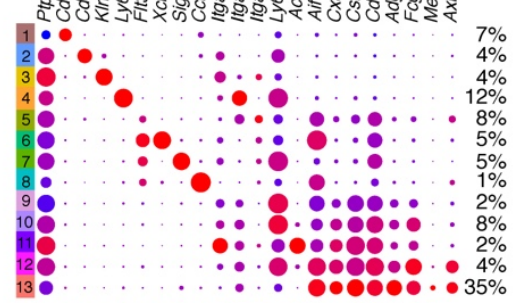

B cells (1) Thells (2)

Monócytes (7) NK cells (3)

$\mathrm{CDC2}(4)$ migDC (6)

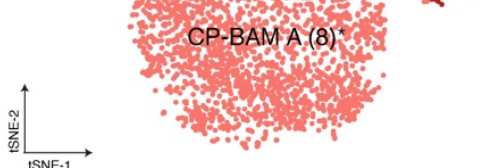

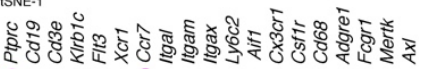

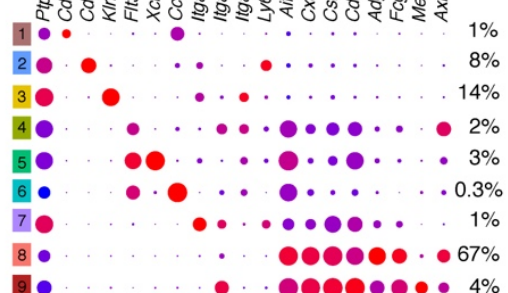

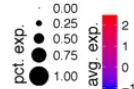

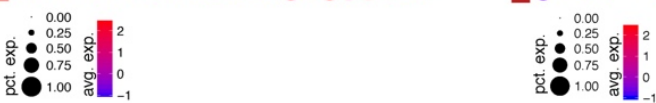

Shared $\mathrm{DC}$ genes $\mathrm{CDC2}$ sig. $\mathrm{CDC} 1$ signature $\quad \mathrm{pDC}$ signature migDC signature

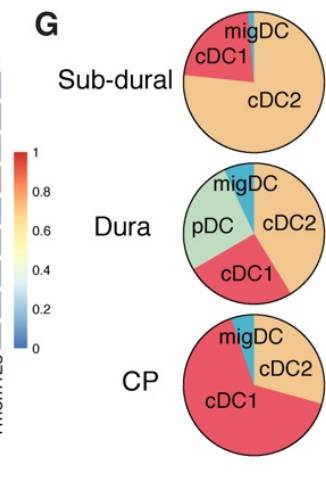



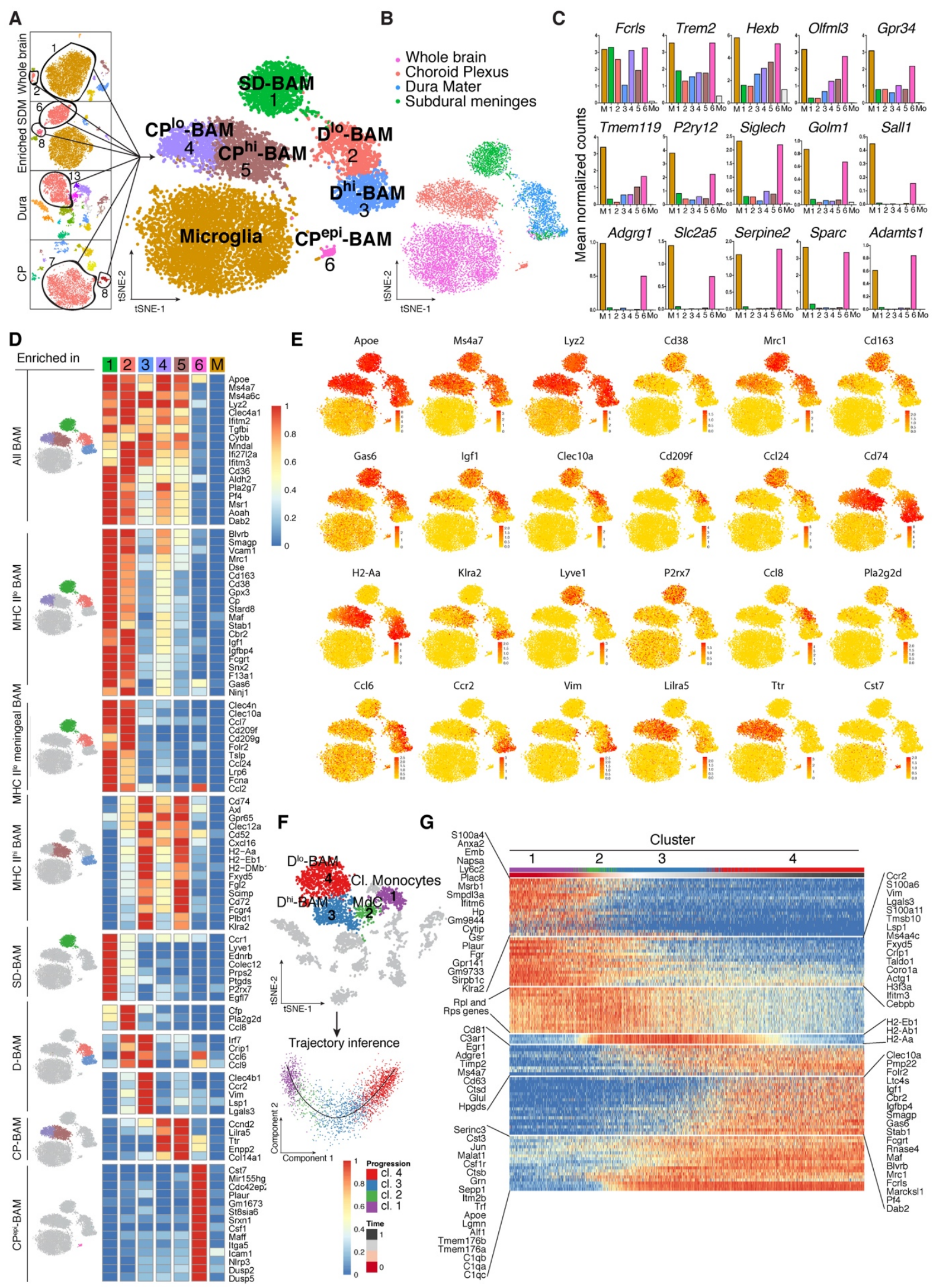

Figure 2. 
A

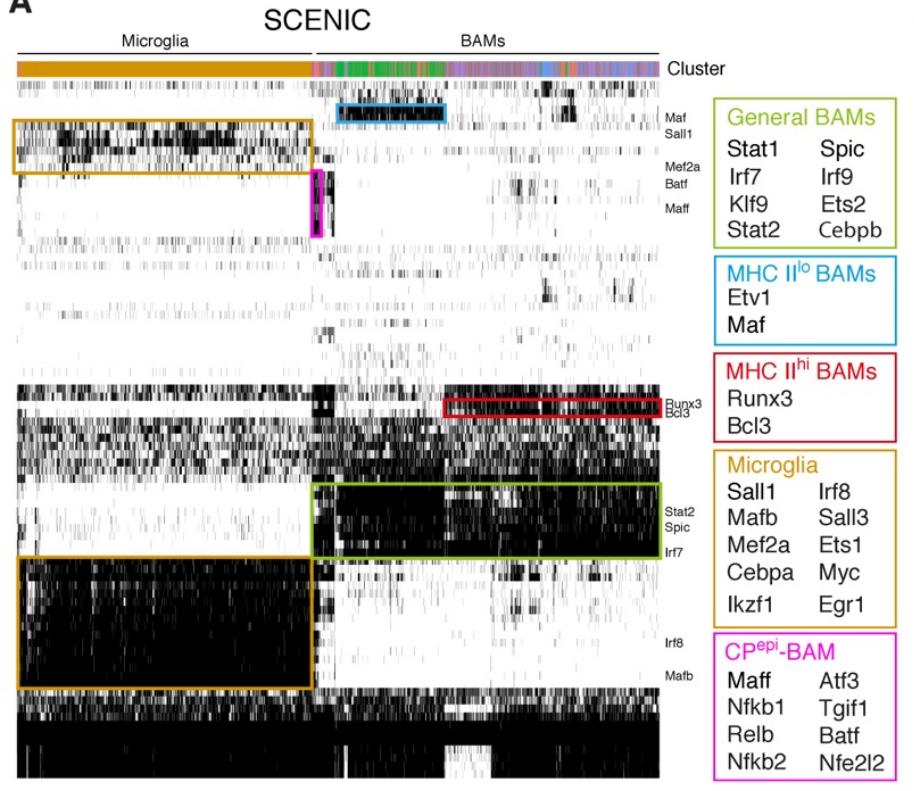

B

tSNE based on expression

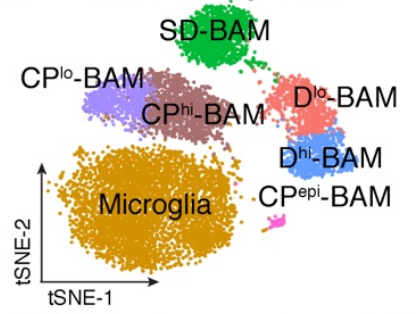

tSNE based on regulon activity

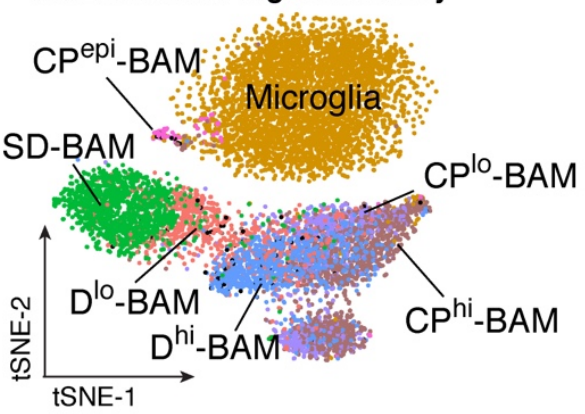

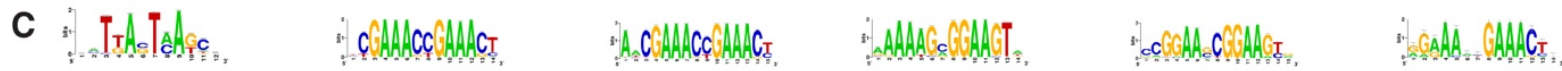
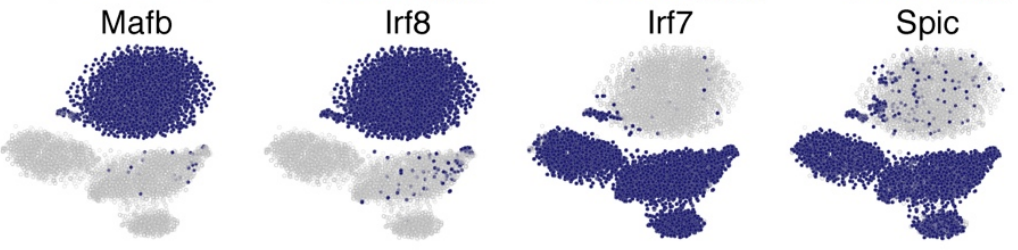

Ets2

Stat1

i.

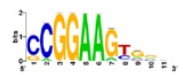

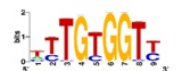

Etv1

Runx3

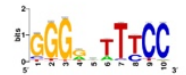

$\mathrm{Bcl} 3$
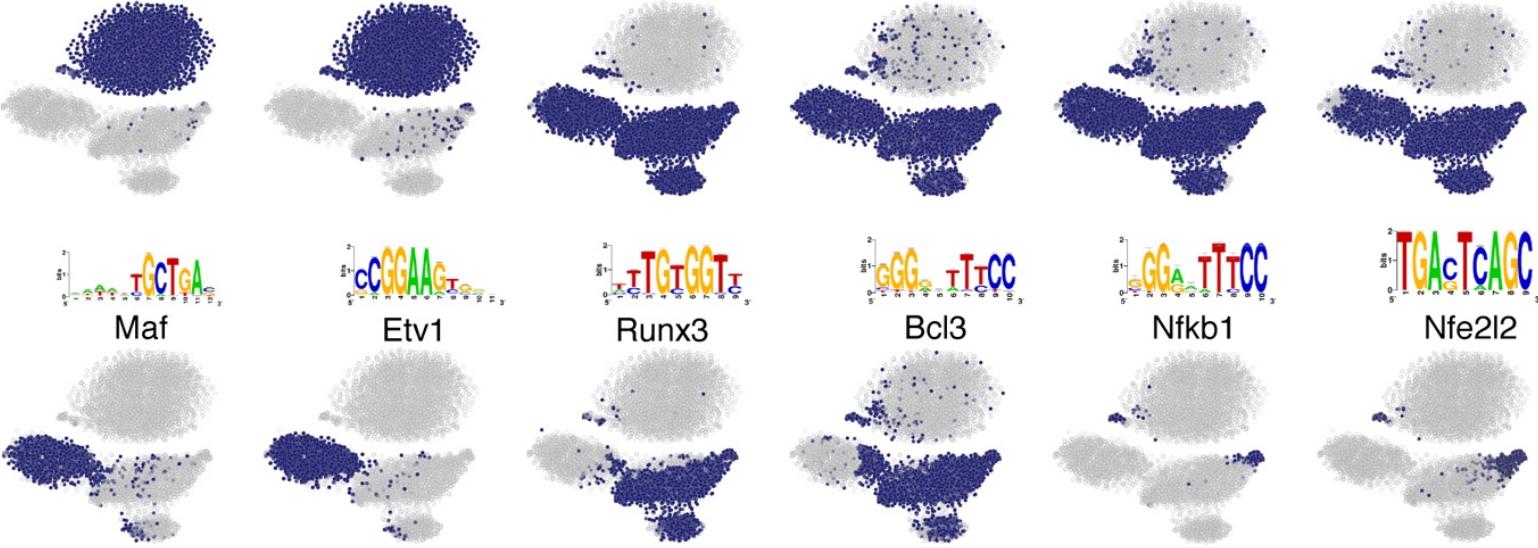

: G. TA TTCC

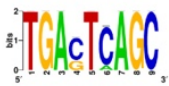

Nfe2l2

Figure 3 

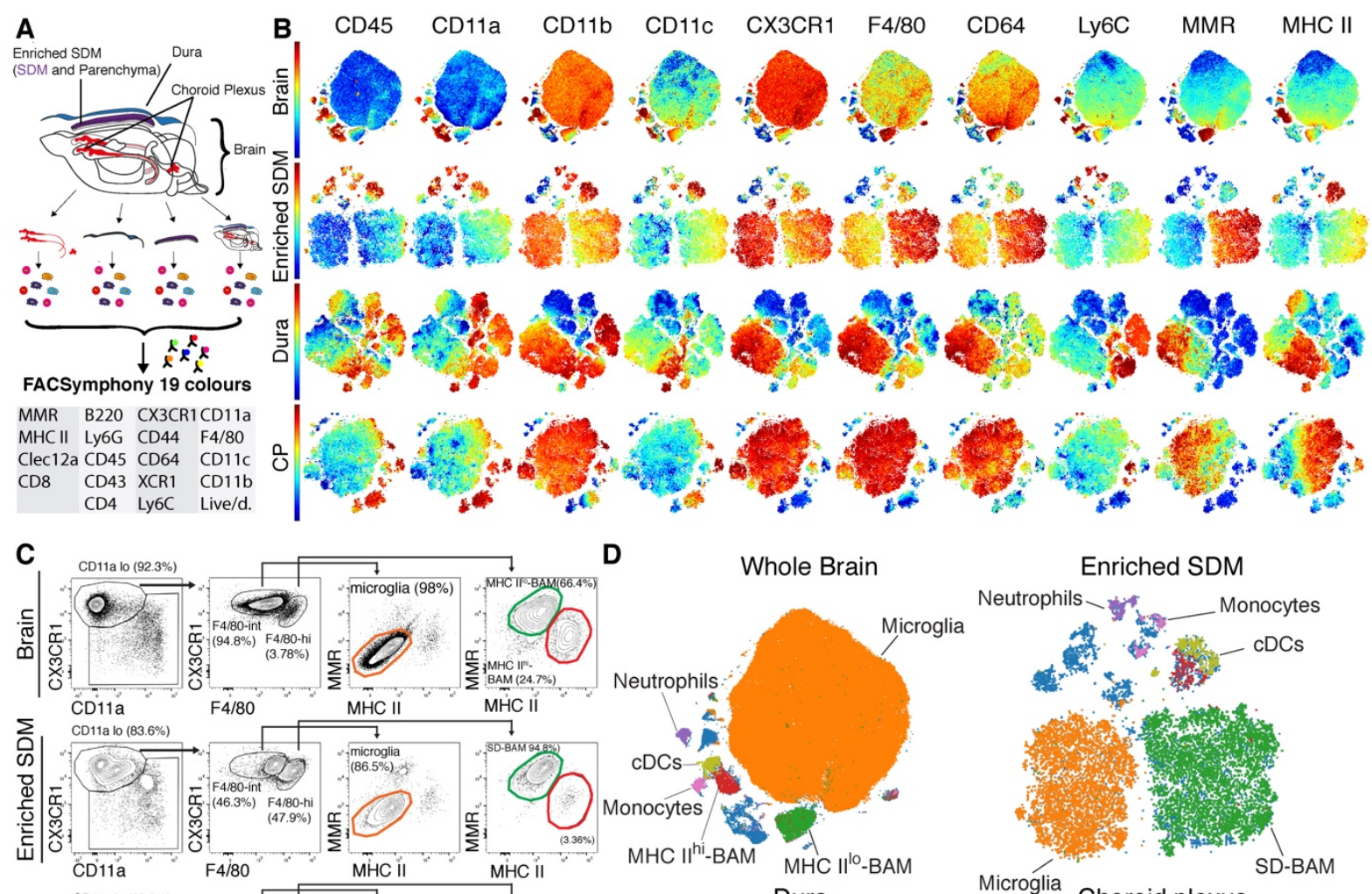

Whole Brain

Enriched SDM
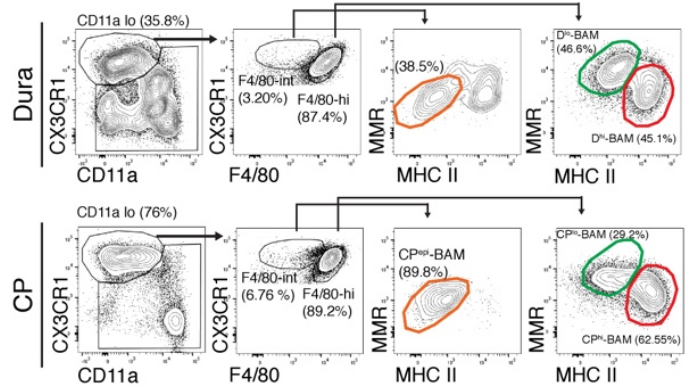

cDCs -

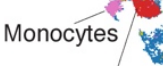

MHC $\|^{\text {hi }}$-BAM MHC $\|^{\text {lo }}-$ BAM

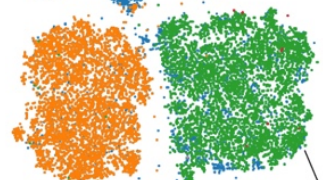
Dura Microglia Choroid plexus

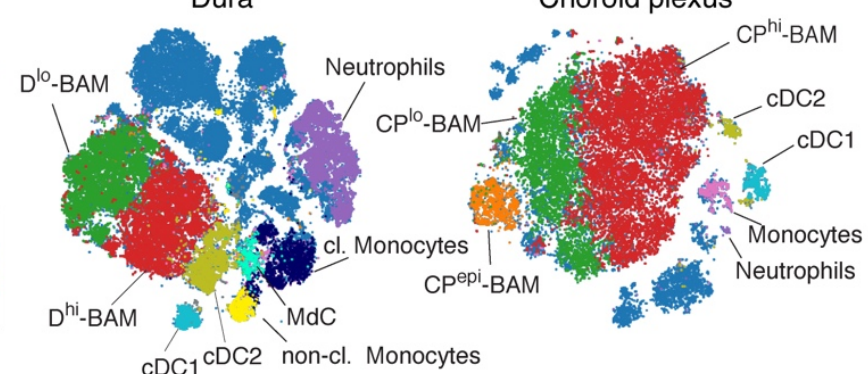

E
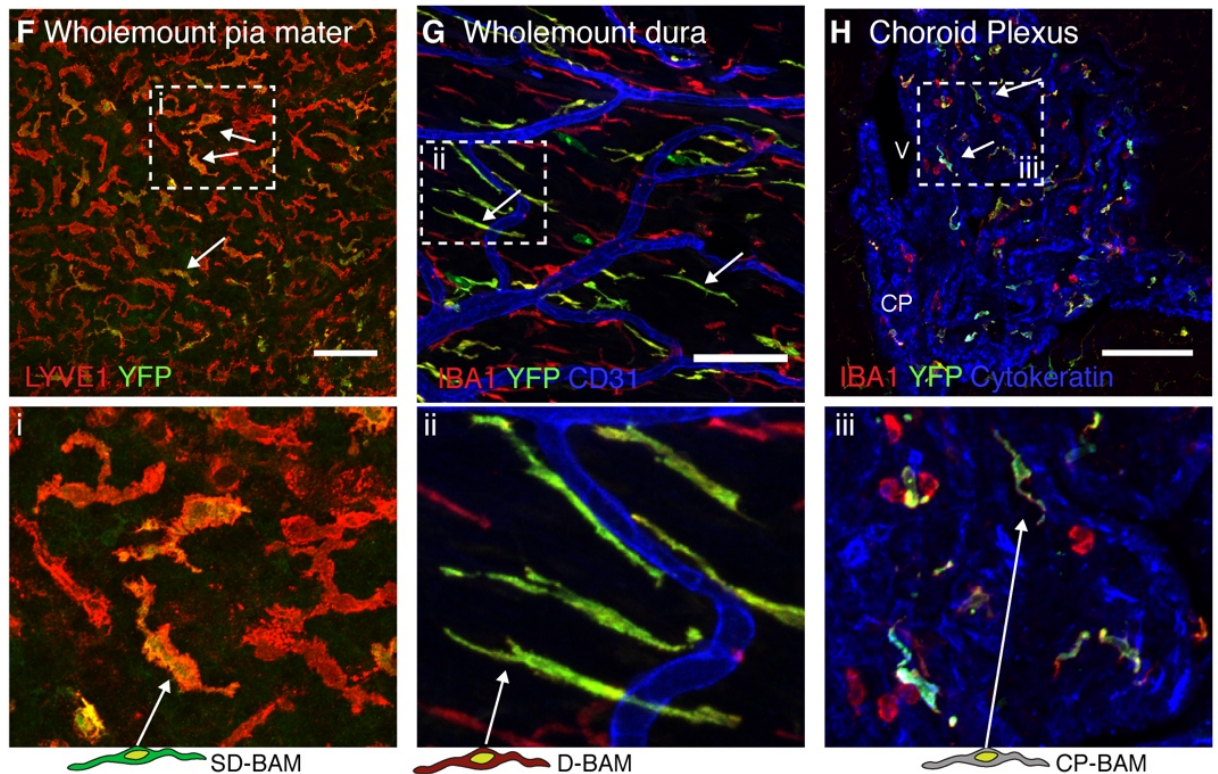

Figure 4. 
A

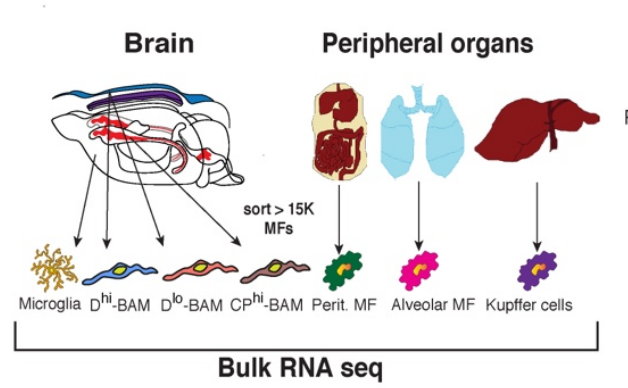

B

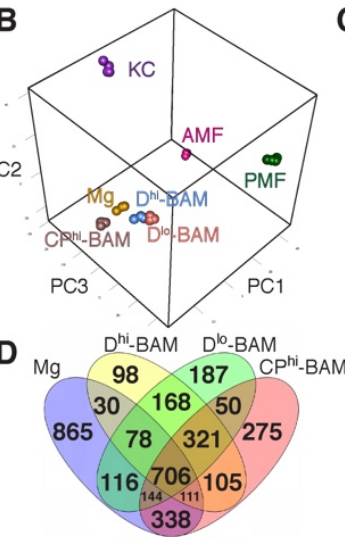

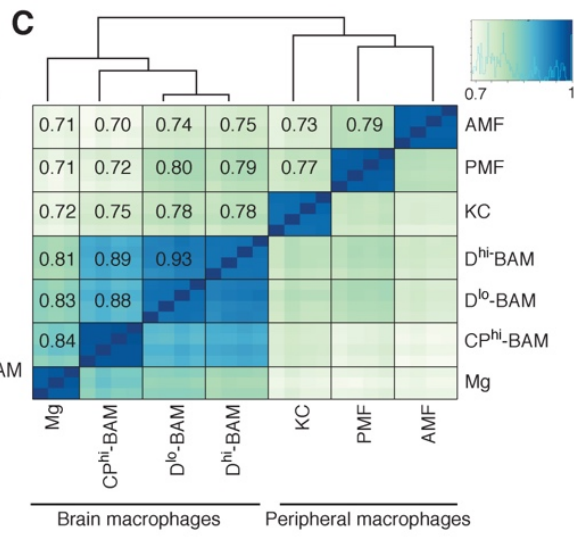

C

Brain macrophages Peripheral macrophages
E

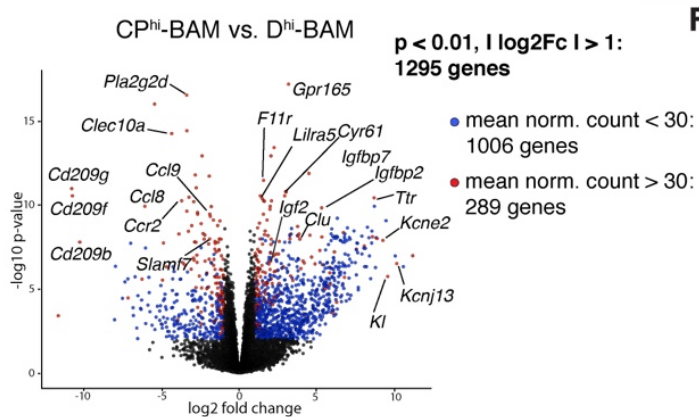

F

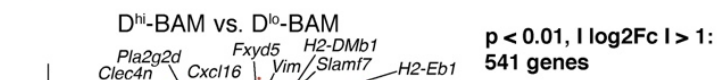

G

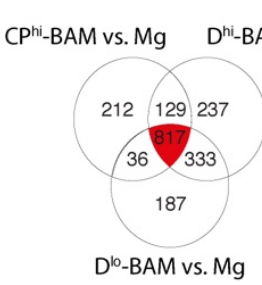

H

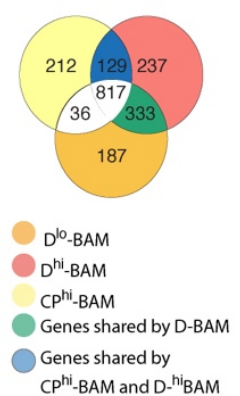

Figure 5.
Regulation of MAPKKK $\log 2$ fold change

$\begin{array}{ll}\text { ignalling cascade } & \text { lipid and alcohol metabolic process } \\ \text { (Lpl, Plbd1, Gdpd5, Cpt1a, Galc, Pcyt1a, Sgpp 1) }\end{array}$

Dusp22, Sorbs3, 111 , Itsn1)
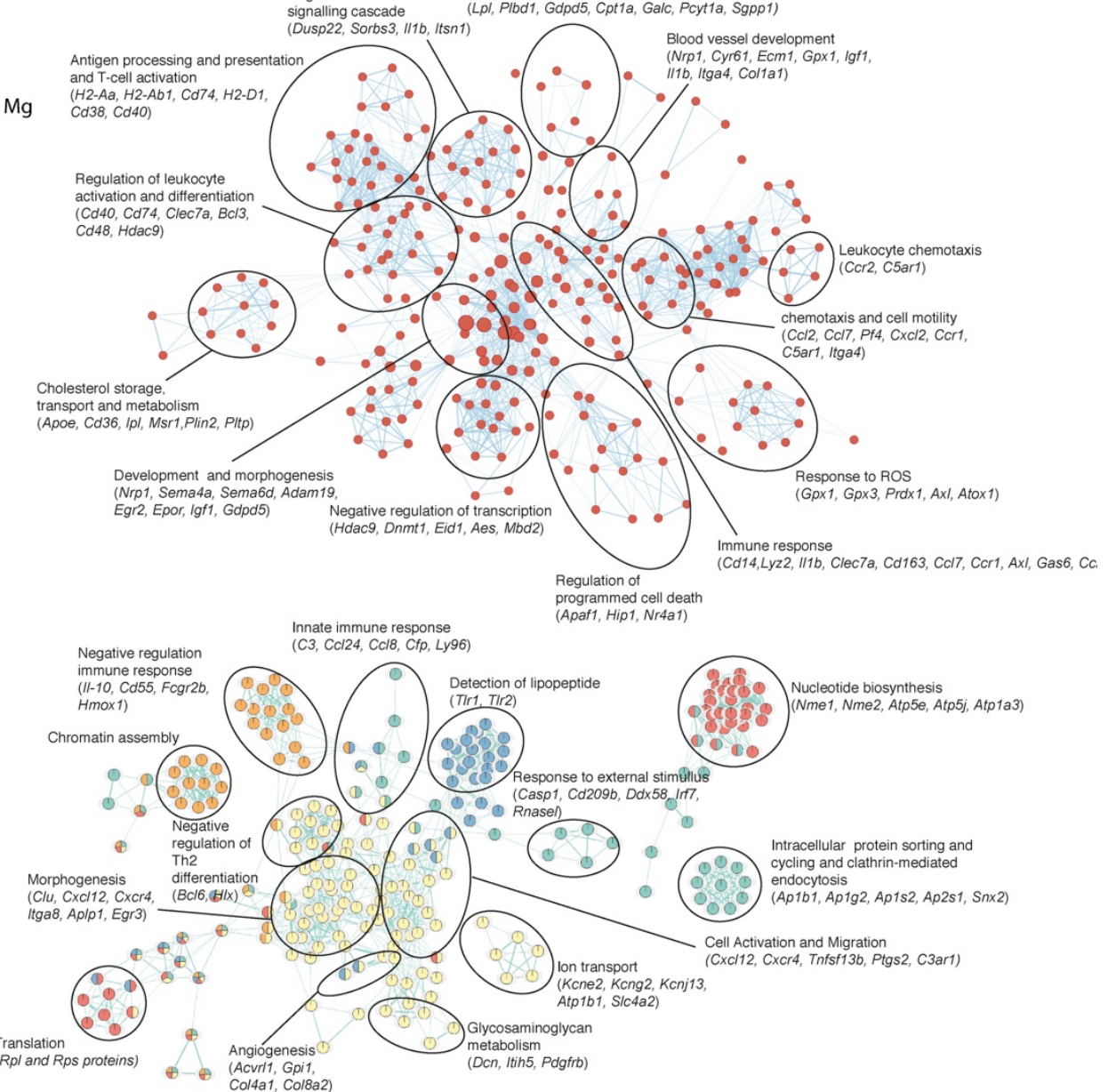
A

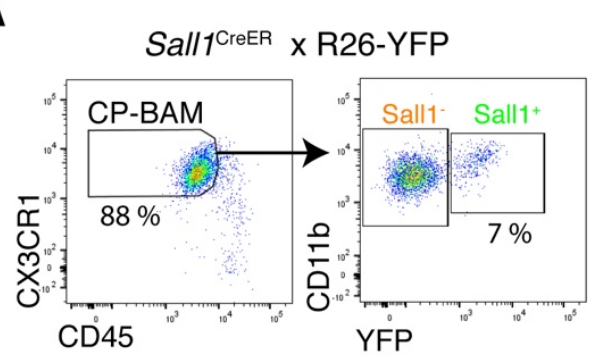

C

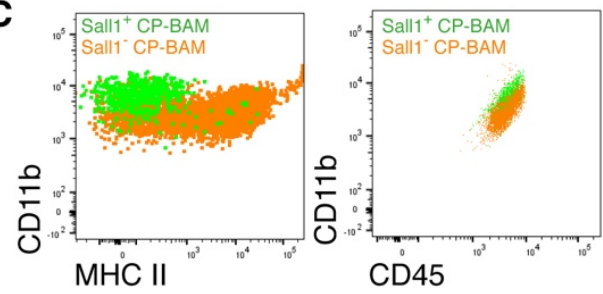

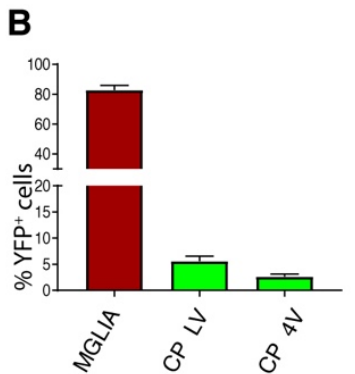

D
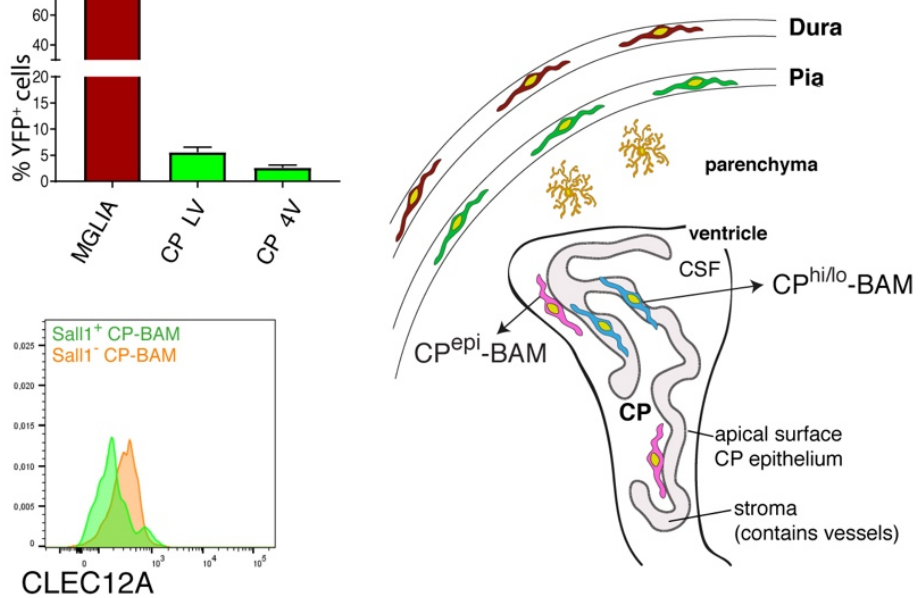

E

Sall $1^{\text {CreER }} \times$ R26-YFP

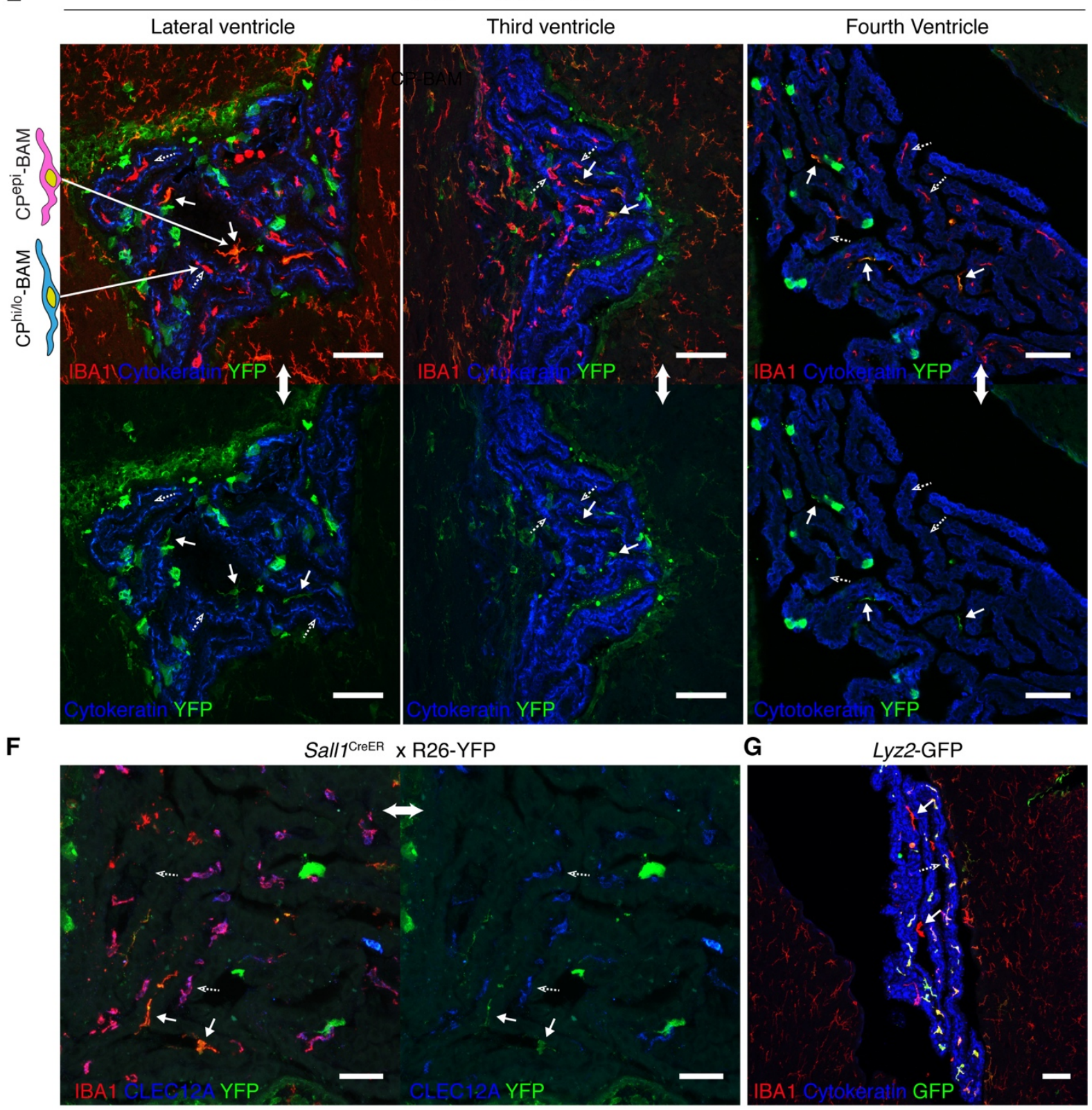

Figure 6.

hird ventricle 
A $\quad \mathrm{CP}^{\mathrm{epi}}$-BAM vs. Microglia B

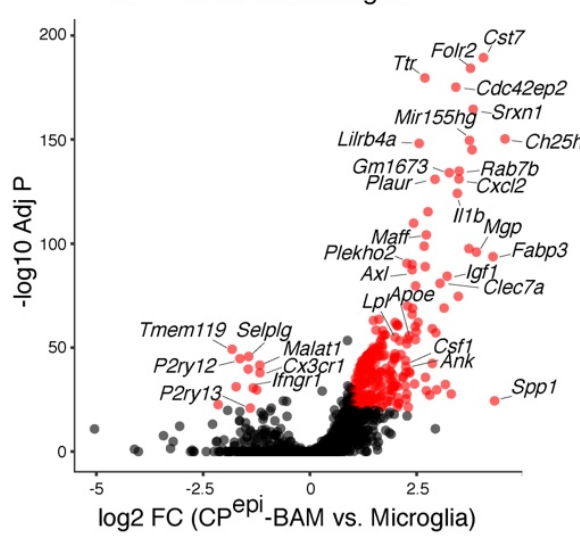

B C

C $\quad$ cst7

D

DAM vs. Microglia

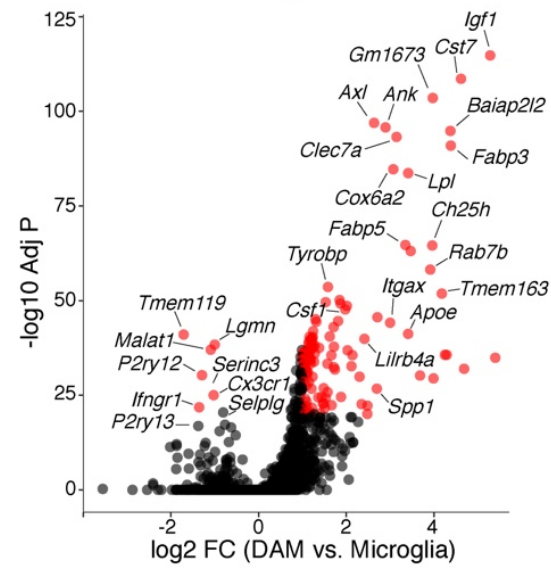

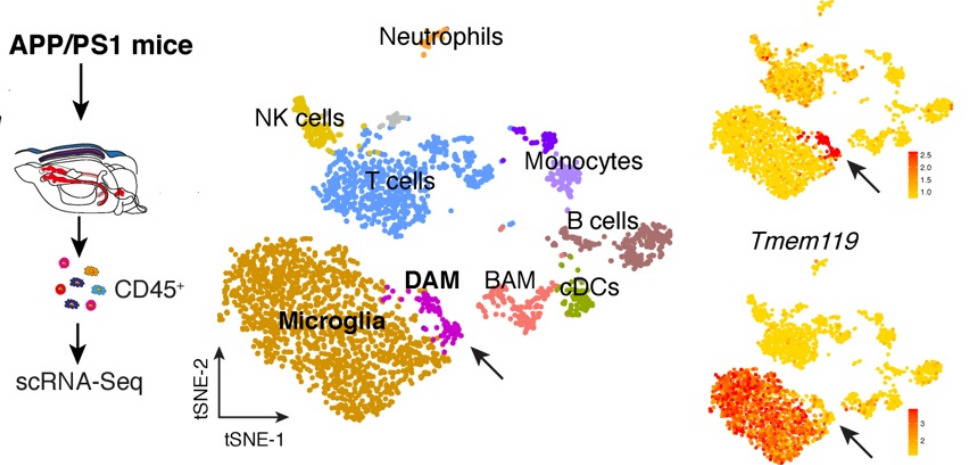

$\mathbf{E}$

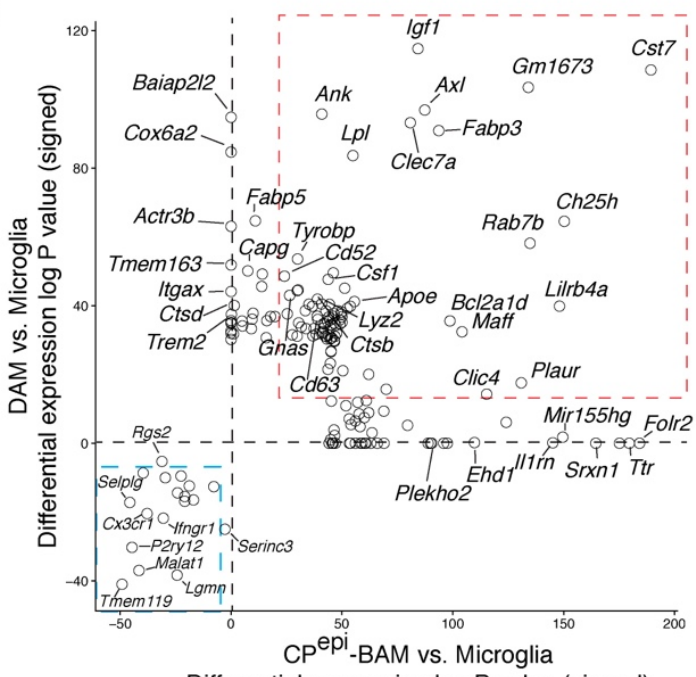

$\mathbf{F}$

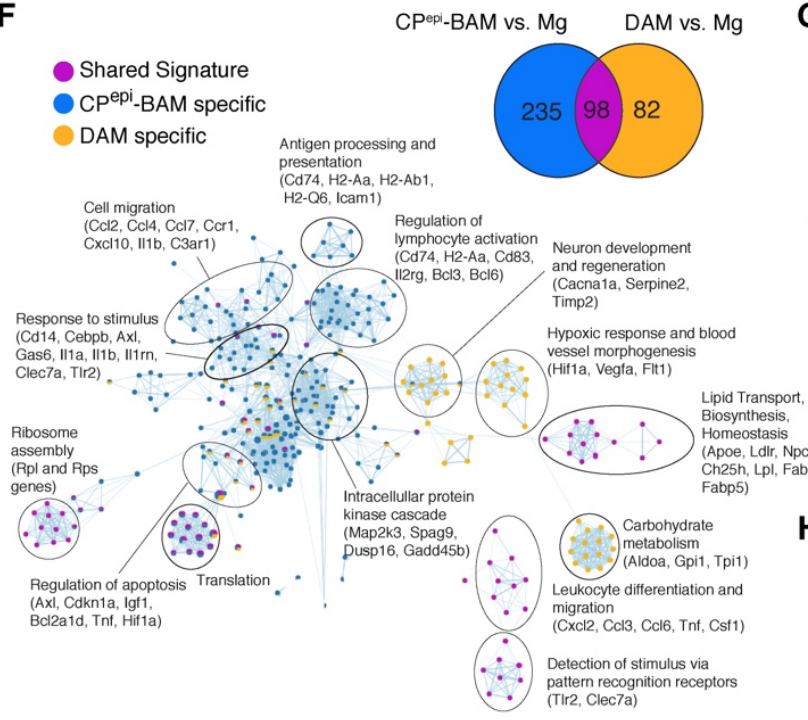

G

Differential expression log $\mathrm{P}$ value (signed)

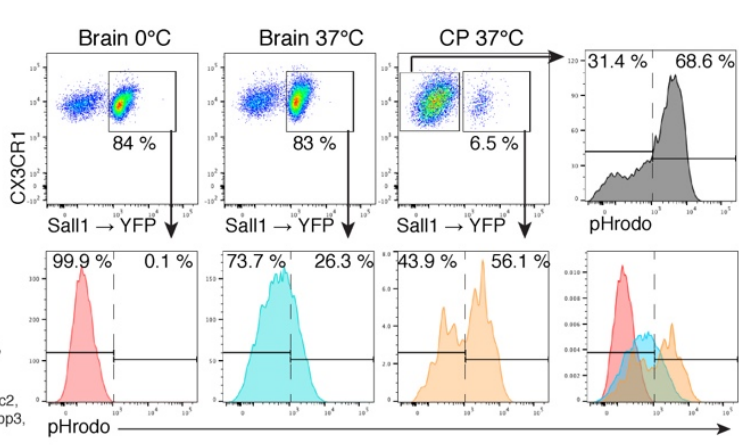

H

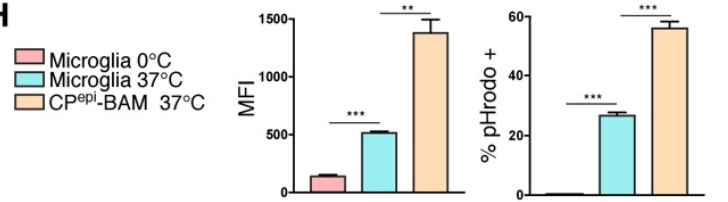

Figure 7. 


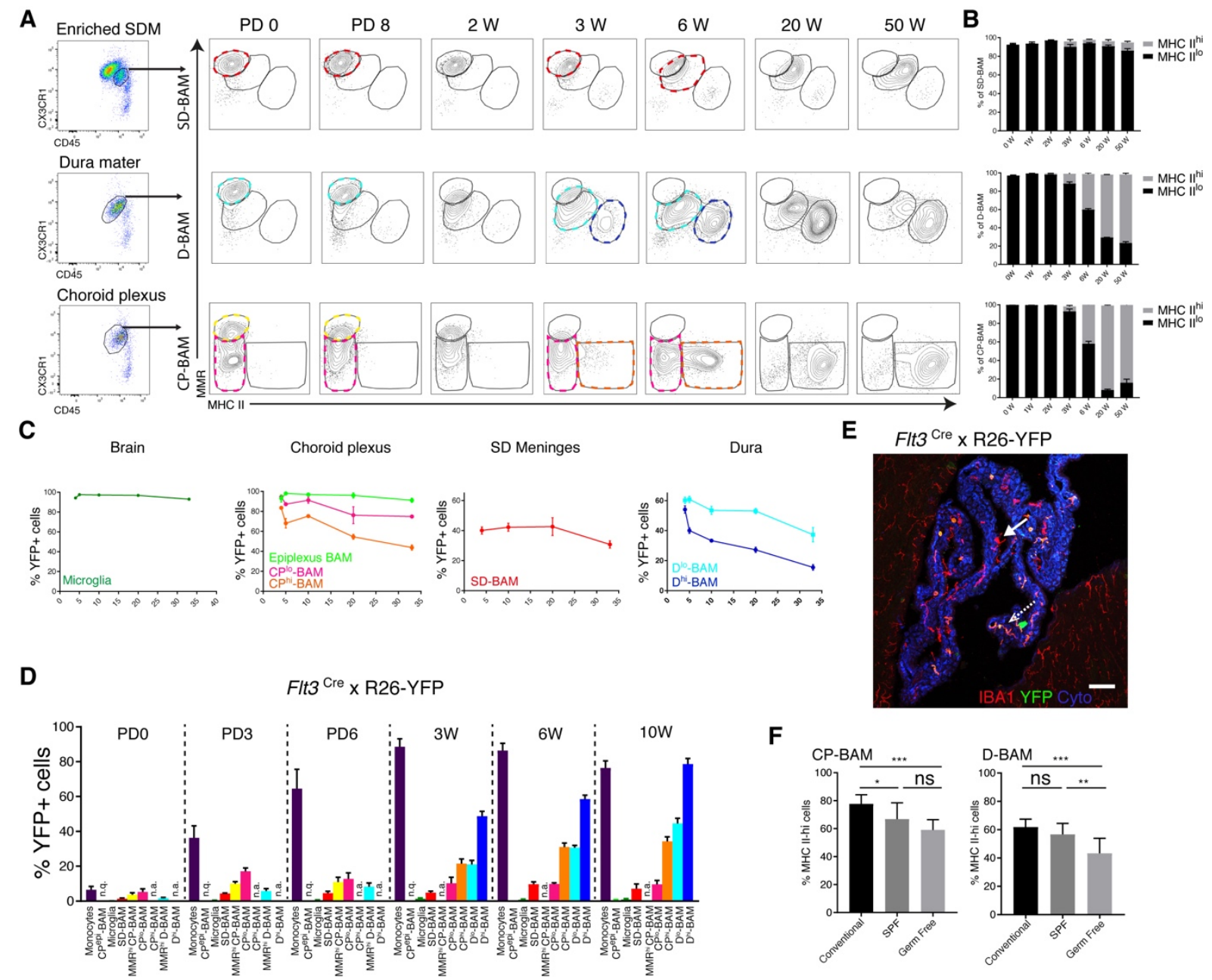

Figure 8. 
Supplementary table 1. Examples of markers genes that were used to identify brain immune cell clusters.

\begin{tabular}{|c|c|}
\hline $\begin{array}{l}\text { Cell type } \\
\text { Myeloid cells }\end{array}$ & \\
\hline Macrophages & Adgre1hi, Fcgr1hi, Csf1hi, Flt3, Ly6c2, Ace- \\
\hline Monocyte-derived cells (MdC) & $\begin{array}{l}\text { Adgre11int, Fcgr1int, Csf1rint, Ly6c2hi, Ccr2hi, Chil3, Ace- } \\
\text { Eno3, } \mathrm{H}^{-}-\mathrm{Aa}^{+}\end{array}$ \\
\hline Classical (cl.) monocytes & $\begin{array}{l}\text { Adgre 1int, Fcgr1lo, Csf1 int, Flt3- Ly6c2hi, Ccr2hi, Chil3+, } \\
\text { Ace- Eno3-, H2-Aa- }\end{array}$ \\
\hline Non-classical (non-cl.) monocytes & 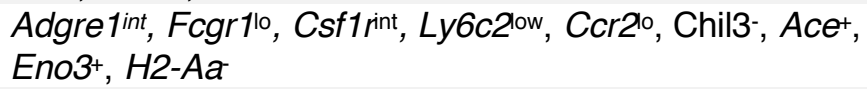 \\
\hline Monocytic cells (MC) & 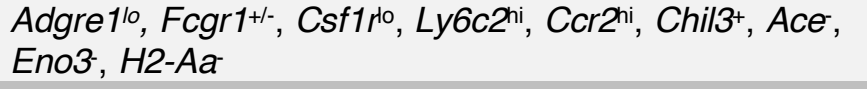 \\
\hline Dendritic cells & \\
\hline $\mathrm{cDC1}$ & Fcgr1-, Flt3+, Itgax+, Itgam, Itgae ${ }^{+}, X{ }^{+} 1^{+}$ \\
\hline cDC2 & 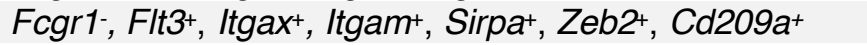 \\
\hline $\mathrm{pDC}$ & Fcgr1-, Flt3 ${ }^{+}$, Itgax + , Siglech+, Ccr9+, Pacsin $1^{+}$ \\
\hline Migratory DC & Fcgr1-, Flt3+, Ccr7+, Nudt17+, Cacnb3 ${ }^{+}$ \\
\hline Granulocytes & \\
\hline Neutrophils & Fcgr1-, Ly6g', $\mathrm{Ngp}^{+}, \mathrm{Camp}^{+}, \mathrm{S100a9^{+ }}$ \\
\hline Lymphocytes & \\
\hline B cells & Cd19+, Pax5+, Ms4a1+ ${ }^{+}$ \\
\hline T cells & $\mathrm{Cd} 3 \mathrm{e}^{+}, \mathrm{Lat}^{+}, \mathrm{Bc} / 11 \mathrm{~b}^{+}$ \\
\hline NKT cells & $\mathrm{Cd}_{3} \mathrm{e}^{+}, \mathrm{KIrb1}^{+}, \mathrm{Ncr}^{+}$ \\
\hline NK cells & Cd3e, Klrb1c $\mathrm{C}^{+} \mathrm{Ncr1}^{+}$, Eomes $^{+}, \mathrm{Gzma}^{+}$ \\
\hline
\end{tabular}


A

Choroid plexus

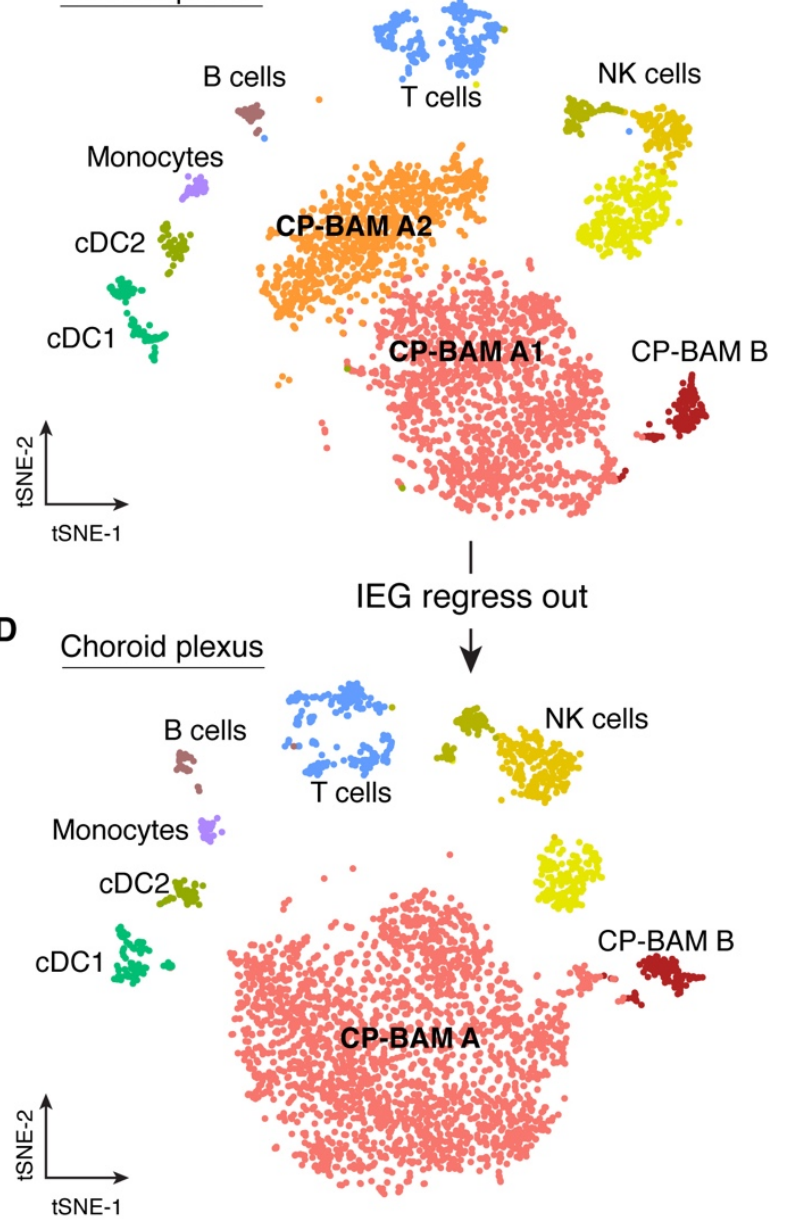

B
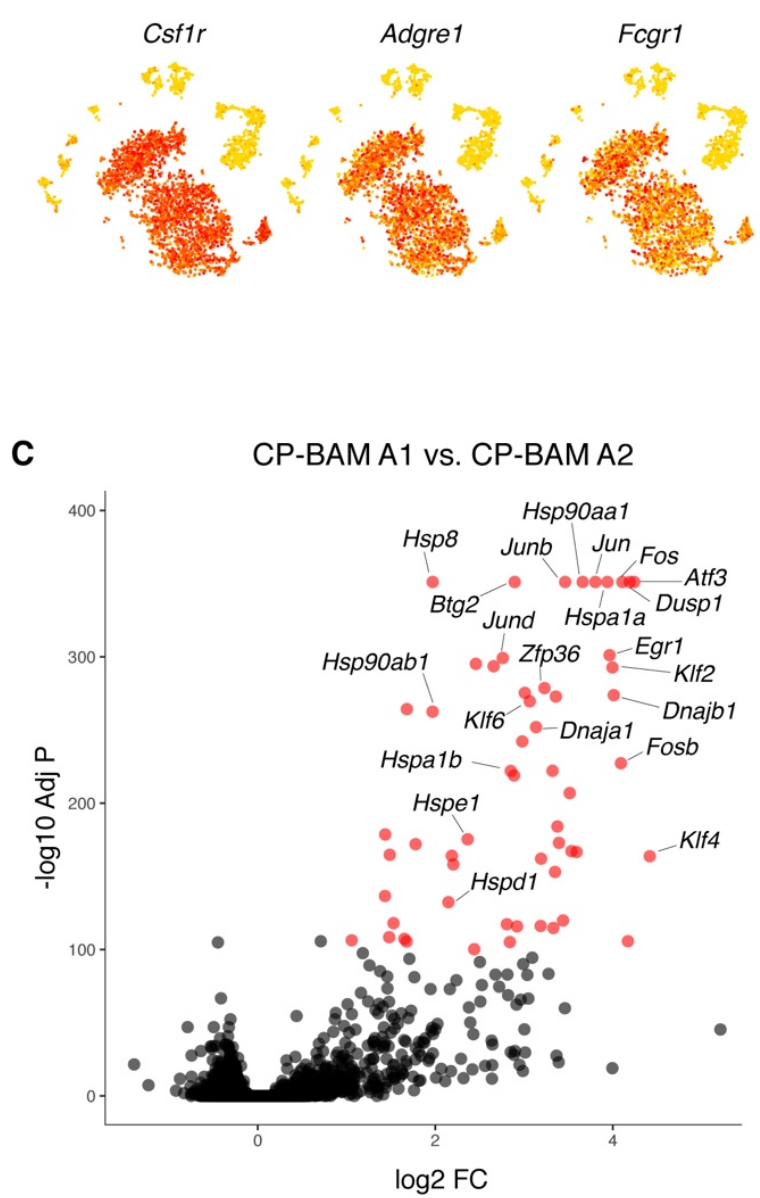

E

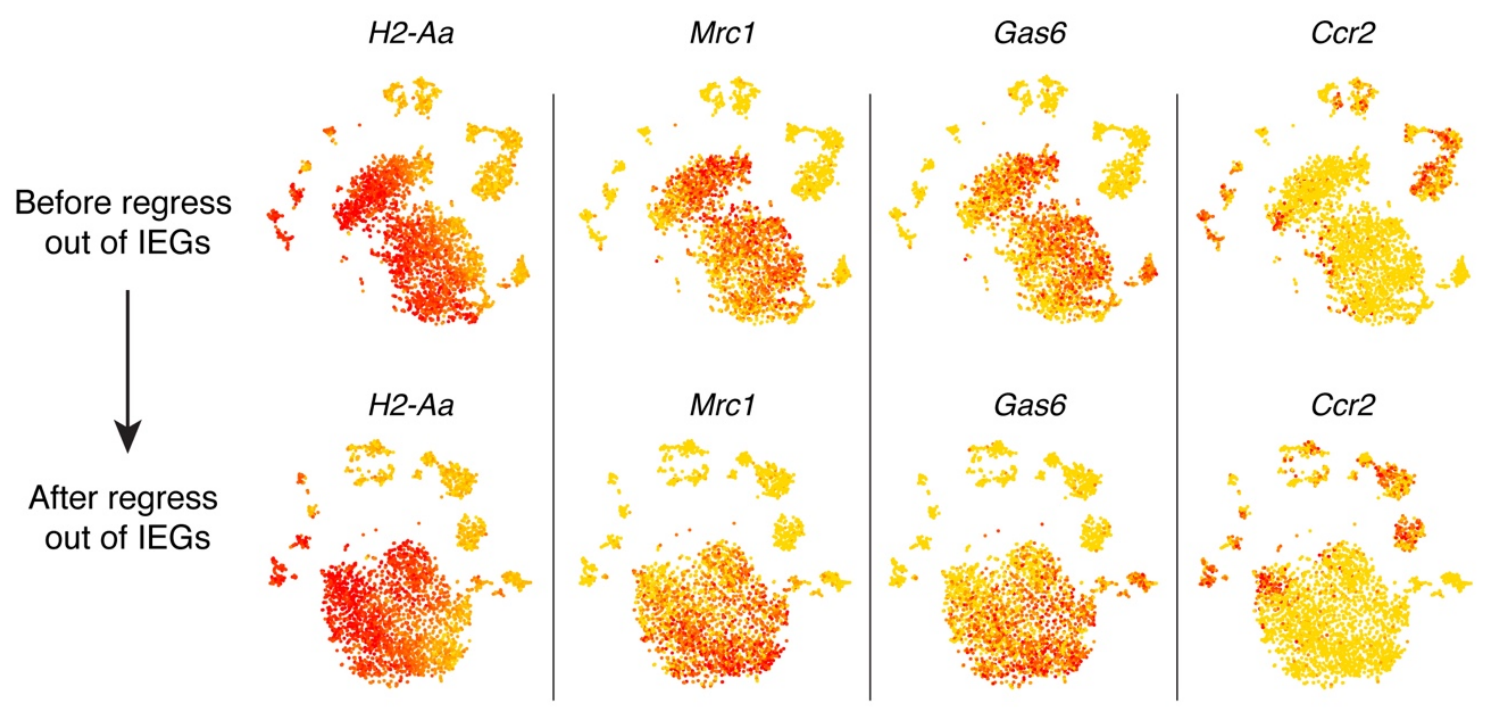

Supplementary Figure 1. 


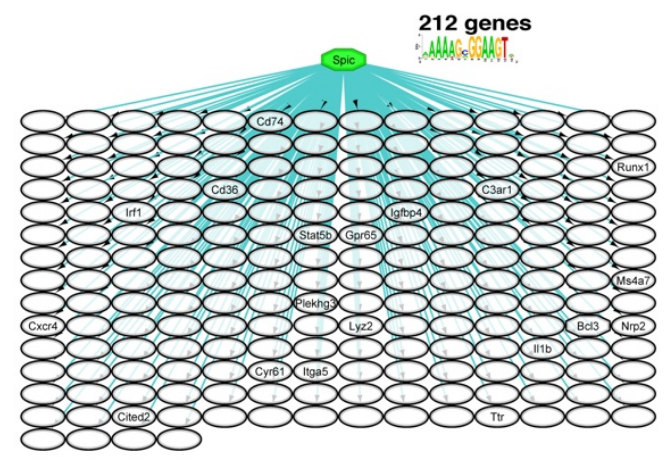

231 genes
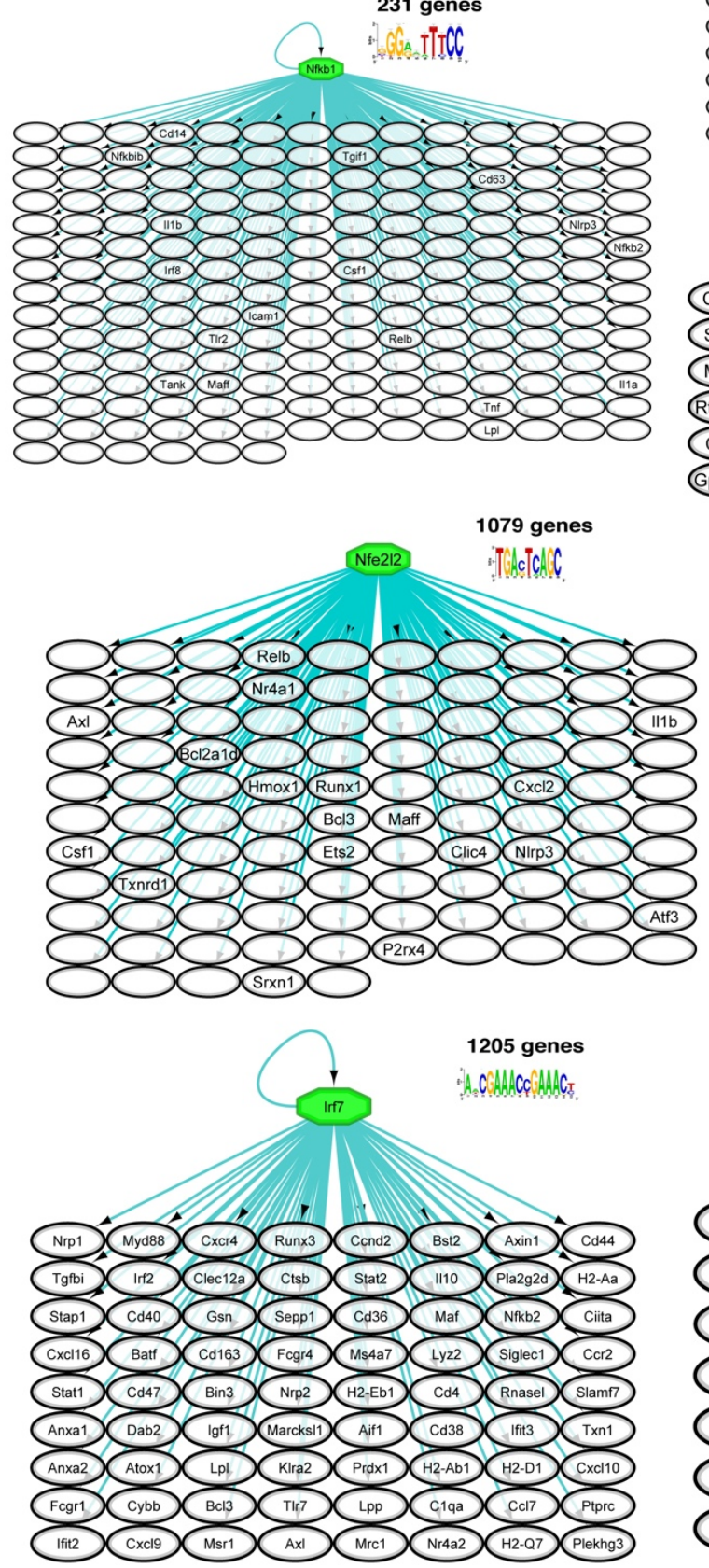
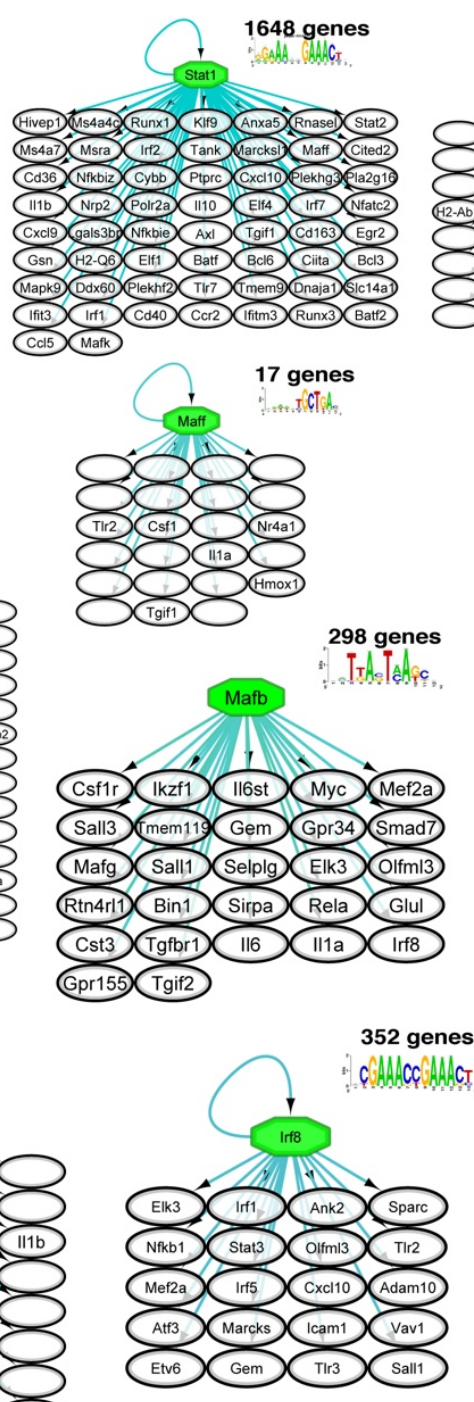
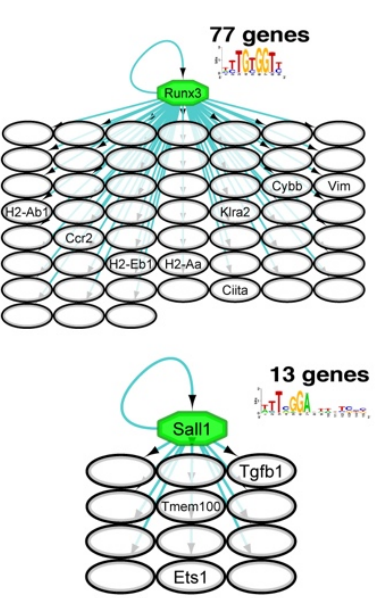

295 genes inf TTCCC<smiles>C1CCCC1</smiles>

(Cd40) (H2-D1) (CD74) Rel10) (12-AD1 Tgiif) (Icam1) (NIPP) (H2-M3 BCD16 (12-Ebi) Maff (1110) Mart Runx1

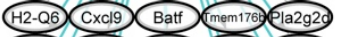
(Is4424) Cxc110 am129 CxC12 (Myd88 Nikb2) (Nikb11) Hivep2) (H2-K1) Stat1 (III) Nitbiip Itigas CCI9 H2-Aa

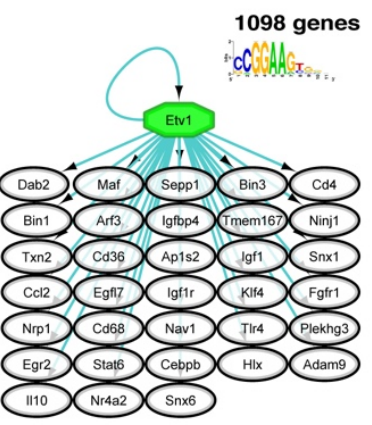

2081 genes

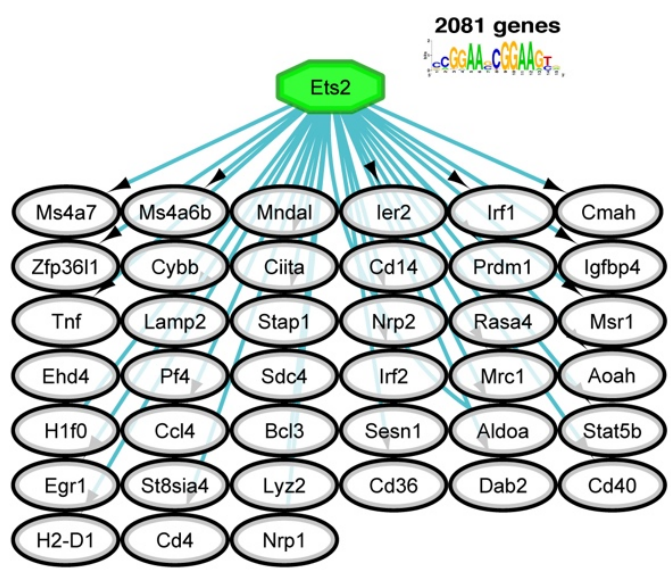

Supplementary Figure 2. 

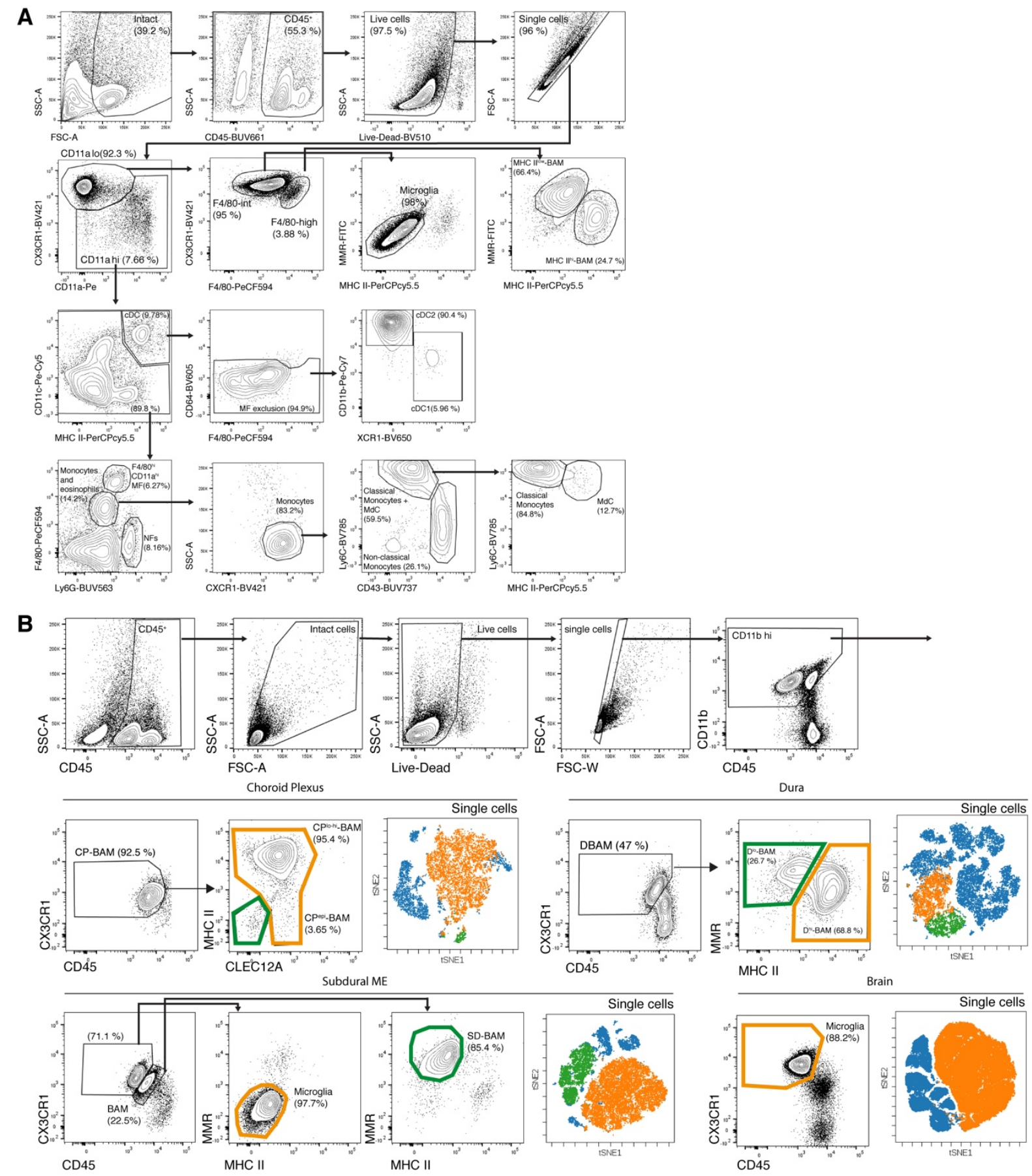

Supplementary Figure 3. 
A

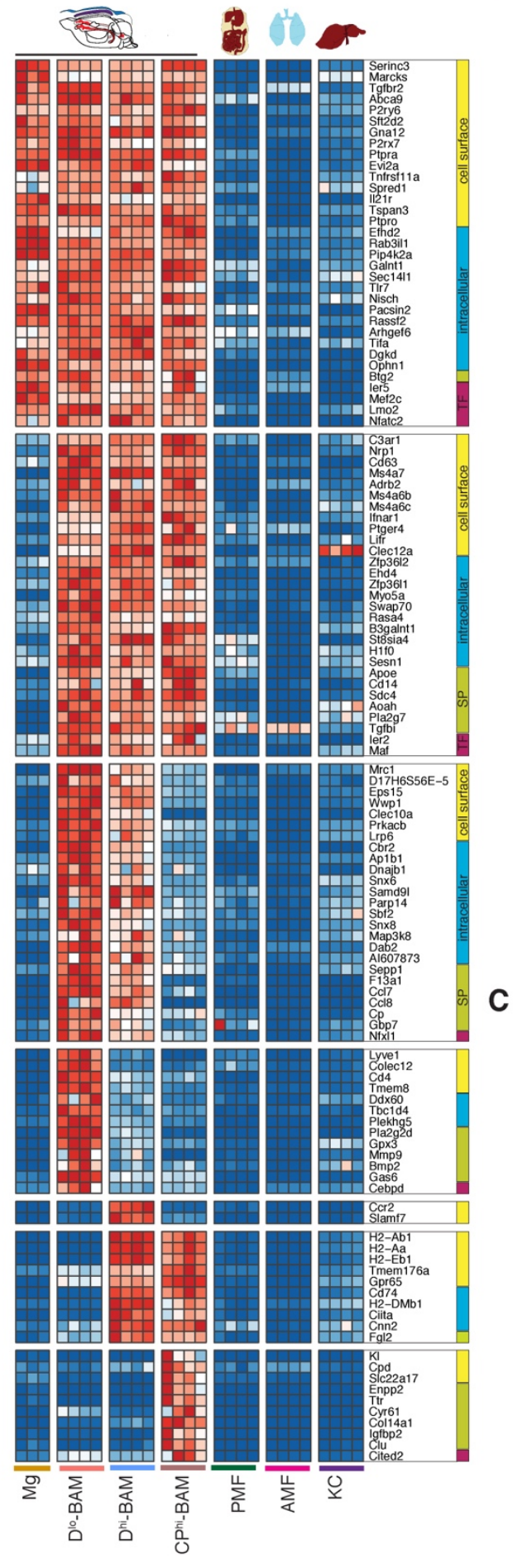

B

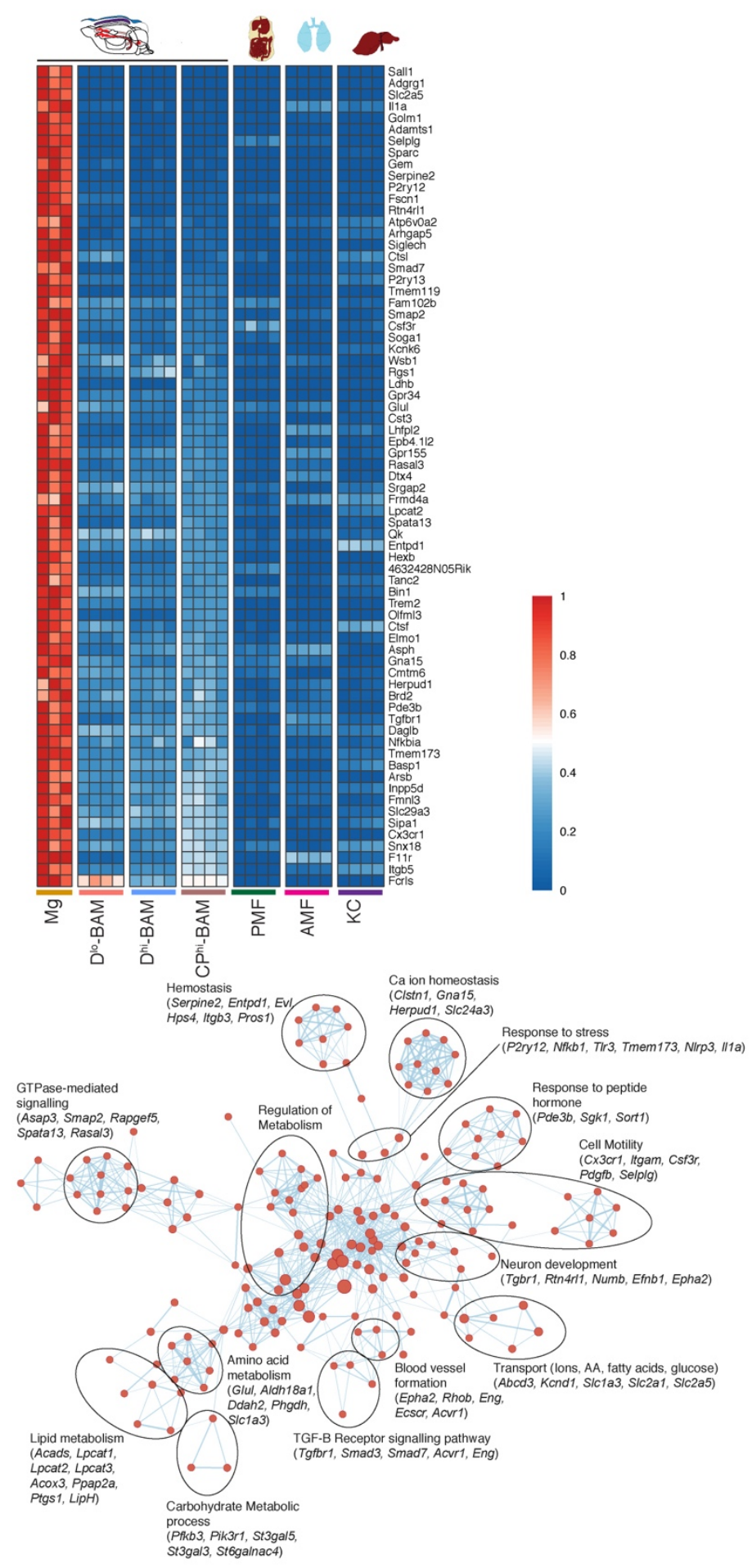

Supplementary Figure 4. 


\section{Materials and Methods}

Mice

C57BL/6J mice were either bred in house or bought from Janvier. Males (Janvier) of 7 weeks and 9 weeks were used for Bulk RNA-sequencing and 10x chromium scRNA-Seq, respectively. For FACSymphony experiments $9 \mathrm{~W}$ old males from Janvier were used. Ten-week-old M/F Germ-Free C57BL/6J mice were bred in the Ghent Germfree and Gnotobiotic mouse facility (Ghent University, Belgium). Germ-Free mice were bred in hepa-filtered flexible-film isolators (North Kent Plastics - NKP). Germ-Free status was routinely monitored by culture-dependent and-independent methods. Cx3cr1CreER 1,2, Flt3Cre 3, Sall1CreER 4, which was kindly provided by Dr. Ryuichi Nishinakamura (Kumamoto University) and R26-YFP 5 mice were bred in house. The sex and ages of the animals used are indicated in the figure legends. Male 9-month-old APP/PS16 mice were used for scRNA-Seq. Mouse experiments were performed in accordance with institutional guidelines and approved by respective governmental authorities.

\section{Whole brain, CP, Dura and Enriched subdural meninges single-cell isolation}

Mice were deeply anaesthetized and perfused transcardially with $30 \mathrm{ml}$ of ice-cold PBS. For whole brain isolations, mice were decapitated, and brains were placed on ice-cold RPMI (Gibco). Brains were cut

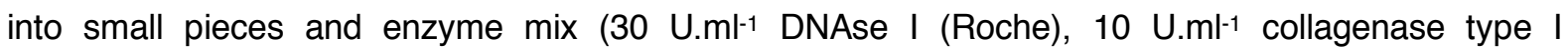

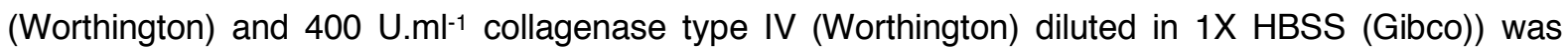
added. Following $20 \mathrm{~min}$ at $37^{\circ} \mathrm{C}$, tissue was crushed with a syringe plunger and homogenized via trituration using standard Serological Pipettes. The solution was filtered twice over a $100 \mu \mathrm{m}$ nylon filter and centrifuged. The pellet was resuspended in $5 \mathrm{ml} \mathrm{70 \%} \mathrm{Standard} \mathrm{Isotonic} \mathrm{Percoll} \mathrm{(SIP)} \mathrm{(GE}$ Healthcare) diluted in 1 X HBSS and gently overlaid with $5 \mathrm{ml}$ of $37 \%$ SIP, followed by a $5 \mathrm{ml}$ layer of $30 \%$ SIP, forming a 3-layered density gradient (centrifuged at $800 \mathrm{~g}, 30 \mathrm{~min}$ without acceleration/breaks). The 70/37\% interphase containing immune cells was collected, centrifuged and resuspended in FACS buffer (2mM EDTA (Duchefa), 2\% heat-inactivated fetal calf serum (Gibco) dissolved in 1X HBSS).

For isolation of the dura mater, the dorsal part of the skull was carefully removed. The Dura was peeled off from the skull cap and placed in ice-cold RPMI. The enriched subdural meninges was isolated by cutting a thin slice of the dorsal cortex using a scalpel. For isolation of the choroid plexi, the ventricles were exposed after which the choroid plexus of the lateral and the fourth ventricle were carefully microdissected and placed in ice-cold RPMI. The choroid plexus, dura and enriched subdural meninges were cut into small pieces and incubated with enzyme mix at $37^{\circ} \mathrm{C}$ for 30 minutes (the solution was resuspended every 10 minutes). Next, solutions were filtered and centrifuged. Cells from the dura and choroid plexus were resuspended in FACS buffer. To remove myelin, cells from the enriched subdural meninges were resuspended in 30\% SIP and centrifuged (2000 rpm, $30 \mathrm{~min}$, no acceleration/brakes) and the pellet was resuspended in FACS buffer. 


\section{Peritoneal macrophage, Alveolar macrophage, Kupffer cell and blood single-cell isolation}

Mice were killed with $\mathrm{CO} 2$ after which a peritoneal lavage was performed with $10 \mathrm{ml}$ ice-cold PBS. The solution was centrifuged $\left(450 \mathrm{~g}, 5 \mathrm{~min}, 4^{\circ} \mathrm{C}\right)$ and the pellet was resuspended in FACS buffer. Alveolar macrophages and Kupffer cells were isolated as described previously? ${ }^{7}$ Blood was isolated either through heart puncture or via the tail vein using heparin-filled syringes. Red blood cells were lysed using lysis buffer (155mM NH4Cl, 10mM KHCO3, 500mM EDTA) followed by neutralization with RPMI and resuspension in FACS buffer.

\section{Single-cell RNA sequencing using 10x Genomics platform}

Single-cell suspensions derived from the dura, choroid plexus, subdural meninges and the whole brain were obtained using above-mentioned procedures. CD45+ immune cells were sorted from each tissue using a BD FACS ARIA ${ }^{\text {TM }}$ III. DAPI (Sigma) was used to exclude dead cells. Sorted cells were centrifuged at $4^{\circ} \mathrm{C}$ at $400 \mathrm{~g}$, resuspended in PBS $+0.04 \%$ bovine serum albumin at $\mathrm{RT}$ at an estimated final concentration of 1000 cells $/ \mu \mathrm{l}$. Cellular suspensions were loaded on a GemCode Single Cell Instrument (10x Genomics, Pleasanton, CA, USA) to generate single-cell Gel Bead-in-Emulsions (GEMs). GEMs and single-cell RNA-Seq libraries were prepared using GemCode Single Cell 3' Gel Bead and Library Kit (Product code: 120237) and the Chromium TM i7 Multiplex Kit (Product code: 120262). Sequencing libraries were loaded on an Illumina HiSeq4000 flow cell with sequencing settings following recommendations of 10x Genomics (Read1: 26 cycles; Read 2: 98 cycles; Index i7: 8 cycles; Index i5: 0 cycles. $2.1 \mathrm{pM}$ loading concentration). All samples had a minimum of 60.000 reads per cell. The Cell Ranger pipeline was designed to perform sample demultiplexing, generate FASTQ files for the Read1, Read2 and i7 sample index. Read2 containing the cDNA was mapped to the reference genome (mouse mm10) using STAR. Subsequent barcode processing, UMI filtering and single-cell 3' gene counting was performed using the Cell Ranger suite and Seurat v2.3. Aggregation of sample conditions was done using the 'Cell Ranger Aggr' software from 10x Genomics. Digital gene expression matrices were preprocessed and filtered using the SCRAN and Scater R packages ${ }^{8}$. Outlier cells were first identified based on 3 metrics (library size, number of expressed genes and mitochondrial proportion) and cells were tagged as outliers when they were 4 median absolute deviation (MADs) away from the median value of each metric across all cells. Secondly, a PCA plot was generated based on 28 metrics in total. Outlier cells in this PCA plot were identified using the R package mvoutlier. Lowabundance genes were removed using the 'calcAverage' function and the proposed workflow. The raw counts were normalised and log2 transformed by first calculating "size factors" that represent the extent to which counts should be scaled in each library. Highly variable genes were detected using the proposed workflow of the scran $\mathrm{R}$ package and by applying FDR $<=0.05 \&$ var.out\$bio $>=0.01$ as cut offs. Unsupervised clustering of the cells was performed and visualized in two-dimensional scatter plots via t-distributed stochastic neighbour embedding (t-SNE) using the Seurat v2.3 R package9.

In the choroid plexus we observed 'dissociation affected' sub-clusters in macrophages by using the workflow proposed by van den Brink et al. ${ }^{10}$. van den Brink et al. provide a list of genes of which the 
expression levels change during the dissociation protocol. We used this gene list to detect cells that are 'dissociation affected' by plotting the expression of all these genes over all cells. When plotting this as a histogram, a clear cut-off can be seen, which splits the cells in 'dissociation affected' and non-affected cells. This information was added to the meta-data attribute of the Seurat object, allowing us to remove this unwanted source of variation using the 'Regress out' function of the Seurat R package.

In all samples, a small cluster could be identified that exhibited the simultaneous expression of both macrophage and lymphocyte genes. These clusters were assumed to be macrophage-lymphocyte aggregates and were excluded from analysis.

We noticed that Clec7a is not present in the mm10 reference dataset provided by $10 x$ Cell Ranger. As our attempt to create a new Cell Ranger mm10 reference dataset with Clec7a included failed, we used Kallisto with default parameters to map the expression of Clec7a. We filtered the SAM output file from Kallisto for the 2 transcripts of Clec7a (ENSMUST00000184581.2 and ENSMUST00000112076.8). The read headers from this filtered SAM file were then mapped to the R1 FASTQ file (Cell Ranger mkfastq output) to get the 26bp read1 (16bp Chromium barcode and 10bp UMI). We extracted the 16bp cell barcode from this $26 \mathrm{bp}$ read and colored these cells on the tSNE plot. To estimate the expression of Clec7a we counted the number of times a cell barcode was present and used these values as raw count input.

\section{Trajectory Inference using SCORPIUS}

SCORPIUS was performed on the classical monocytes (Cl. Monocytes), Monocyte-derived cells (MdC), $D^{\text {hi-BAM }}$ and Dlo-BAM clusters of the dura mater sample. We started from the log2 normalized counts (via scran R package), selected the clusters of interest and performed SCORPIUS using the proposed workflow ${ }^{11}$.

\section{Single-cell regulatory network inference and clustering using SCENIC}

We performed SCENIC ${ }^{12}$ by starting from the raw counts and by following the proposed workflow using the default parameters. The co-expression network was generated using GENIE3 instead of GRNBoost2. The regulons were visualized in a network using iRegulon 1.313 in Cytoscape 3.5.1.14.

\section{Flow cytometry and FACSymphony}

After obtaining a single cell suspension, live-dead staining was performed using Zombie Aqua Fixable Live-dead (BioLegend) following the manufacturer's protocol. Subsequently cells were blocked with rat anti-mouse CD16/CD32 (clone: 2.4G2) for $15 \mathrm{~min}$ on ice and stained for $20 \mathrm{~min}$ using the antibodies listed in supplementary table 2. After staining, cells were washed with FACS buffer. The FACSymphony experiments were performed three independent times and were analyzed using the $B D$ FACSymphony ${ }^{\mathrm{TM}}$ A5. Prior to acquisition, the PMT voltages were adjusted to minimalize fluorescence spillover. Single-stain controls were prepared with UltraComp eBeads ${ }^{\mathrm{TM}}$ (Thermofisher) following manufacturer's instructions and were used to calculate a compensation matrix. Other flow cytometry experiments were performed using the BD FACS Canto ${ }^{\mathrm{TM}}$ II. 
Flow cytometry data was analyzed using FlowJo_V10 and Cytobank (https://www.cytobank.org).

\section{Flow cytometry statistics}

All graphs represent mean \pm SEM. The difference between two means in the phagocytosis assay (Fig. $7 \mathrm{H}$ ) and the germ-free experiment (Fig. $8 \mathrm{~F}$ ) was analyzed using the two-tailed unpaired student's t-test in Graphpad Prism 7.03. The decline in the percentage of YFP+ cells (Fig. 8C) and the increase in the percentage of YFP+ cells (Fig. 8D) was evaluated using a ONE-WAY ANOVA in IBM SPSS Statistics.

\section{Bulk RNA-sequencing}

The different macrophage populations were isolated using the above-mentioned procedures. The single cell suspensions were blocked with anti-mouse CD16/CD32 (clone: 2.4G2) and stained. 7-AAD (Biolegend) was used to exclude dead cells. For CPhi-BAMs, between 14000 and 18000 cells were sorted four independent times. For Dhi-BAMs and D lo-BAMs $9000-18000$ and $7000-15000$ cells were sorted 4 independent times, respectively. 30000 microglia were sorted 3 independent times. 30.000 peritoneal MFs and 25.000 alveolar and Kupffer cells were sorted 4 independent times. Samples were sorted in $500 \mu \mathrm{l}$ Buffer RLT (Qiagen RNeasy Plus Micro Kit) containing 1\% 2-mercaptoethanol (SigmaAldrich). Cell sorting was performed using the BD FACS ARIA ${ }^{\mathrm{TM}}$ II. RNA was isolated using the Qiagen RNeasy Plus Micro Kit following manufacturer's instruction. RNA concentration and purity were determined spectrophotometrically using the Nanodrop ND-1000 (Nanodrop Technologies) and RNA integrity was assessed using a Bioanalyser 2100 (Agilent). Per sample, an amount of $1 \mathrm{ng}$ of total RNA was used as input for the SMART-Seq v4 Ultra Low Input RNA protocol (version "040215") from Clontech Laboratories, Inc. Subsequently, 1ng of purified cDNA was sheared to $300 \mathrm{bp}$ using the Covaris M220. From the sheared material, sequencing libraries were prepared with the NEBNext Ultra DNA Library Prep Kit for Illumina (version 2.0 -1/15), according to the manufacturer's protocol including a size selection to $250 \mathrm{bp}$ insert size. Sequence-libraries of each sample were finally equimolarly pooled and sequenced on NextSeq500 flow-cell: High Output, 75 bp, Single Reads, v2.

\section{Bulk RNA-sequencing data analysis}

The preprocessing of the RNA sequencing data was done by Trimmomatic. The adapters were cut off, and reads were trimmed when the quality dropped below 20 . Reads with a length $<35$ were discarded. All samples passed quality control based on the results of FastQC. Reads were mapped to the mouse reference genome (mm10) via Tophat2 and counted via HTSeqCount. Samples were subsequently analyzed using R/Bioconductor, and the $R$ package limma was used to normalize the data and to perform differential expression (DE) analysis. For analysis of DE genes, we applied a stringency level where the adjusted $p$-value was equal to 0.05 and the log2FC was less than -1 or greater than 1 . A list of core genes for a specific sample or a group of samples was generated by first calculating the $D E$ genes between the sample(s) of interest and all the other samples. This list of DE genes was then filtered for the genes that showed a mean and median difference $>1$ between the sample(s) of interest and all the other samples. Then the expression of the resulting genes was transformed into values 
between 0 and 1. Only the genes that follow the desired expression profile - e.g. normalized expression values $<0.5$ for all samples, except for the sample(s) of interest, there the values should be $>0.5$ were kept. Finally, we also removed all genes with normalized counts $<100$.

\section{Functional analysis using gene ontology enrichment analysis}

To predict putative biological functions based on DE genes, we carried out a Gene Ontology Enrichment analysis. Genes that were differentially expressed (adjusted $p$-value $<0.01$ ) between microglia and the Dlo-BAM, Dhi-BAM and CPhi-BAM subsets were used to determine lists of the shared and BAM subsetspecific genes (see Venn diagram Fig. 5G). All genes with at least a two-fold change in expression (log2FC >1) were selected (CPhi-BAM: 129, Dhi-BAM: 151, Dlo-BAM: 112, shared CPhi-BAM and DhiBAM: 70, shared Dhi-BAM and Dlo-BAM: 190) and inserted into BINGO 3.0.3.15 to obtain the enriched ontologies using the standard settings. An enrichment map was produced based on these ontologies using EnrichmentMap v3.1.0.16 (FDR q-value: 0.05, p-value: 0.001, Jaccard: 0.25) and AutoAnnotate v1.2.17 in Cytoscape 3.5.114. The color of the nodes represents the BAM subset or shared BAM subset to which they belong. The 385 genes with a $\log 2 \mathrm{FC}>1$ and the 432 genes with a $\log 2 \mathrm{FC}<-1$ that were shared between all the BAM subsets were used to produce two additional enrichment maps. The gene ontology enrichment map of the DAM and CPepi-BAMs was produced starting from the genes that were differentially expressed between both subsets and microglia (-logPadj $>10)$. The genes with a log2FC $>1$ from each subset were selected. The CPepi-BAM-specific genes (235), DAM-specific genes (82) and the genes shared between these two subsets (98) were used to produce an enrichment map. The color of the nodes corresponds to the subset.

\section{Tamoxifen treatment}

3-4-week-old Cx3cr1CreER $\times$ R26-YFP and Sall1CreER $\times$ R26-YFP mice were treated with $4 \mathrm{mg}$ tamoxifen (Sigma-Aldrich) dissolved in $200 \mu \mathrm{l}$ cornoil (Sigma-Aldrich), which was injected subcutaneously near the fore and hind limbs $(4 \times 50 \mu \mathrm{l})$. These injections were repeated three times at 48 -hour intervals.

\section{Immunohistochemistry of brain sections and wholemounts}

Mice were deeply anaesthetized and transcardially perfused with ice-cold PBS followed by $4 \%$ ice-cold paraformaldehyde (PFA)/PBS solution. Brains were carefully removed and post-fixed in 4\% PFA/PBS at $4^{\circ} \mathrm{C}$ for 4 hours. Brains were dehydrated in $15 \%$ sucrose/PBS $(0 / n)$ followed by $30 \%$ sucrose/PBS $(\mathrm{o} / \mathrm{n})$ and embedded in Tissue-Tek O.C. ${ }^{\mathrm{TM}}$ compound (Sakura Finetek Europe B.V. Alphen aan den Rijn, NL) and frozen using dry ice. $12 \mu \mathrm{M}$ cryosections were made using a Leica CM $1850 \mathrm{UV}$. Sections were blocked and permeabilized using $0.1 \%$ PBS-Triton ${ }^{\mathrm{TM}} \mathrm{X}-100$ (PBS-T) (Sigma-Aldrich) containing $10 \%$ Normal Donkey Serum (NDS) (v/v) (Sigma) for 1 hour. Sections were incubated with primary antibodies (diluted in a PBS-T solution containing $3 \%$ NDS) $\mathrm{o} / \mathrm{n}$ at $4^{\circ} \mathrm{C}$ in a moist chamber. The following primary antibodies were used: chicken anti-GFP (id. Ab13970, polyclonal, lotnr. GR3190550-3, Abcam, 1/250), mouse anti-cytokeratin (id. Ab7753, clone C-11, lotnr. GR3190550-3, Abcam, 1/5000), rabbit anti-IBA1 (id. 019-19741, polyclonal, lotnr. WDE1198, Wacko, 1/500) and rat anti-CLEC12a (id. 
143402, clone 5D3/Clec12a, lotnr. B151240, BioLegend, 1/10). Secondary antibodies were incubated for 1.5 hour at RT. The following secondary antibodies were used: Goat anti-chicken Alexa Fluor 488 (id. A11039, polyclonal, lotnr. 1691381, Life Technologies, 1/500), Goat anti-rat Alexa Fluor 555 (id. A21434, polyclonal, lotnr. 1722994, Life Technologies, 1/500), Donkey anti-rabbit Alexa Fluor 647 (id. A31573, polyclonal, lotnr. 1693297, Life Technologies, 1/500), Goat anti-mouse Alexa Fluor 546 (id. A21123, polyclonal, lotnr. 1830291, Invitrogen, 1/500). Samples were mounted using mowiol (Polysciences Inc.) mounting medium.

Dura wholemounts were obtained by removing the dura from the skull cap after PFA perfusion. Wholemounts of the enriched SDM were obtained by cutting a thin slice from the pineal gland to the posterior part of the olfactory bulb. Wholemounts were post-fixed on ice-cold 4\% PFA for 2-2.5 hours after which they were washed with PBS $(2 \times 3 \mathrm{~min})$ at RT. Wholemounts were blocked and permeabilized in $0.1 \%$ (Dura) or $0.3 \%$ (Pia) PBS-T containing 10\% NDS (v/v) for 1.5 hour. Primary antibodies were diluted in a $3 \%$ NDS PBS-T solution and left to incubate $o / n$ at $4^{\circ} \mathrm{C}$ in a moist chamber. The following primary antibodies were used: rat anti-Lyve1 (id. 14-0443-82, clone ALY7, lotnr. 4316105, BD biosciences, 1/100), rat anti-CD31 (id. 550274, clone MEC 13.3, lotnr. 5162734, BD biosciences, 1/100), rabbit anti-Iba1 (id. 019-19741, polyclonal, lotnr. WDE1198, Wacko, 1/500), chicken anti-GFP (id. Ab13970, polyclonal, lotnr. GR3190550-3, Abcam, 1/250). Secondary antibodies were diluted in a $3 \%$ NDS $0.1 \%$ or $0.3 \%$ PBS-T solution and incubated for $1.5 \mathrm{~h}$ : Goat anti-chicken Alexa Fluor 488 (id. A11039, polyclonal, lotnr. 1691381, Life Technologies, 1/500), Goat anti-rat Alexa Fluor 555 (id. A21434, polyclonal, lotnr. 1722994, Life Technologies, 1/500) and Donkey anti-rabbit Alexa Fluor 647 (id. A31573, polyclonal, lotnr. 1693297, Life Technologies, 1/50). Wholemounts were mounted using mowiol mounting medium. Images were taken using the Zeiss LSM880 Fast Airy scan or the Zeiss LSM710. Images were analyzed using Fiji 18.

\section{Phagocytosis assay}

Single-cell suspensions were prepared from the choroid plexus and brain of Sall1CreER $x$ R26-YFP mice following the above-mentioned procedures. Samples were left on ice for $30 \mathrm{~min}$ to slow down cell metabolism. Subsequently, cells were resuspended in $100 \mu \mathrm{L}$ of pHrodo $^{\mathrm{TM}} \mathrm{E}$. coli BioParticles ${ }^{\mathrm{TM}}$ conjugate for phagocytosis (Thermofisher) reconstituted in $2 \mathrm{ml} / \mathrm{vial}$ of RPMI supplemented with $10 \%$

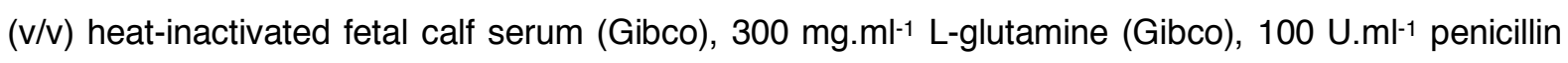
and $100 \mathrm{mg}^{\mathrm{ml}} \mathrm{l}^{-1}$ streptomycin (Gibco). Cells were incubated for 1.5 hours at $37^{\circ} \mathrm{C}$ to allow uptake of particles.

\section{Data availability}

All single-cell RNA-seq and bulk RNA-seq data are deposited under accession code xxxxx. 


\section{References materials and methods}

1. Goldmann, T. et al. A new type of microglia gene targeting shows TAK1 to be pivotal in CNS autoimmune inflammation. Nat. Neurosci. 16, 1618-1626 (2013).

2. Yona, S. et al. Fate Mapping Reveals Origins and Dynamics of Monocytes and Tissue Macrophages under Homeostasis. Immunity 38, 79-91 (2013).

3. Benz, C., Martins, V. C., Radtke, F. \& Bleul, C. C. The stream of precursors that colonizes the thymus proceeds selectively through the early $T$ lineage precursor stage of $T$ cell development. J. Exp. Med. 205, 1187-1199 (2008).

4. Inoue, S., Inoue, M., Fujimura, S. \& Nishinakamura, R. A mouse line expressing Sall1-driven inducible cre recombinase in the kidney mesenchyme. Genesis 48, 207-212 (2010).

5. Madisen, L. et al. A robust and high-throughput Cre Repooting and characterization. Nat Neurosci 13, 133-140 (2010).

6. Radde, R. et al. A 442 -driven cerebral amyloidosis in transgenic mice reveals early and robust pathology. EMBO Rep. 7, 940-946 (2006).

7. Scott, C. L. et al. The Transcription factor Zeb2 is required to maintain tissue-specific identities of macrophages. Immunity (2018) In Press.

8. Lun, A. T. L., McCarthy, D. J. \& Marioni, J. C. A step-by-step workflow for low-level analysis of single-cell RNA-seq data with Bioconductor. F1000Research 5, 2122 (2016).

9. Satija, R., Farrell, J. A., Gennert, D., Schier, A. F. \& Regev, A. Spatial reconstruction of singlecell gene expression data. Nat. Biotechnol. 33, 495-502 (2015).

10. van den Brink, S. C. et al. Single-cell sequencing reveals dissociation-induced gene expression in tissue subpopulations. Nat. Methods 14, 935-936 (2017).

11. Cannoodt, R., Saelens, W., Sichien, D. \& Tavernier, S. SCORPIUS improves trajectory inference and identifies novel modules in dendritic cell development. bioRxiv 079509 (2016). doi:https://doi.org/10.1101/079509

12. Aibar, S. et al. SCENIC: Single-cell regulatory network inference and clustering. Nat. Methods 14, 1083-1086 (2017).

13. Janky, R. et al. iRegulon: From a Gene List to a Gene Regulatory Network Using Large Motif and Track Collections. PLoS Comput. Biol. 10, e1003731 (2014).

14. Shannon, P. et al. Cytoscape: a software environment for integrated models of biomolecular interaction networks. Genome Res. 2498-2504 (2003). doi:10.1101/gr.1239303.metabolite

15. Maere, S., Heymans, K. \& Kuiper, M. BiNGO: A Cytoscape plugin to assess overrepresentation of Gene Ontology categories in Biological Networks. Bioinformatics 21, 3448-3449 (2005).

16. Merico, D., Isserlin, R., Stueker, O., Emili, A. \& Bader, G. D. Enrichment map: A networkbased method for gene-set enrichment visualization and interpretation. PLoS One 5, (2010).

17. Kucera, M., Isserlin, R., Arkhangorodsky, A. \& Bader, G. D. AutoAnnotate: A Cytoscape app for summarizing networks with semantic annotations. F1000Research 5, 1717 (2016).

18. Schindelin, J. et al. Fiji: an open-source platform for biological-image analysis. Nat. Methods 9, 676-682 (2012). 
Supplementary Table 2: Antibodies used in flow cytometry experiments: Antigen, Fluorophore, Clone, Lot number, Manufacturer.

\begin{tabular}{|c|c|c|c|c|}
\hline Antigen & Fluorophore & Clone & Lot number & Manufacturer \\
\hline CD206 (MMR) 1 & FITC & C068C2 & B213957 & BioLegend \\
\hline Clec12a 1 & APC & 5D3 & B188838 & BioLegend \\
\hline CD241 & Alexa Fluor 700 & M1/69 & B223302 & BioLegend \\
\hline CD8a 1 & APC/cy 7 & $53-6.7$ & B217171 & BioLegend \\
\hline CD441 & BV570 & IM7 & B237222 & BioLegend \\
\hline CD641 & BV605 & X54-5/7.1 & B240410 & BioLegend \\
\hline Xcr11 & BV650 & ZET & B241219 & BioLegend \\
\hline Ly-6C1 & BV785 & HK1.4 & B237063 & BioLegend \\
\hline CD45R/B2201 & BUV496 & RA3-6B2 & 7018902 & BD Horizon \\
\hline Ly6G $^{1}$ & BUV563 & $1 \mathrm{~A} 8$ & 7097851 & BD Horizon \\
\hline CD451 & BUV661 & $30-\mathrm{F} 11$ & 7207676 & BD Horizon \\
\hline CD43 1 & BUV737 & S7 & 7143811 & BD Horizon \\
\hline CD41 & BUV805 & GK1.5 & 7125755 & BD Horizon \\
\hline F4/801 & PE-CF594 & T45-2342 & 7241543 & BD Horizon \\
\hline CD11c $^{1}$ & PE/cy5 & N418 & B195382 & Biolegend \\
\hline CX3CR11 & BV421 & SA011F11 & B231871 & Biolegend \\
\hline CD11b1 & PE/cy7 & $\mathrm{M} 1 / 70$ & B227804 & Biolegend \\
\hline $\mathrm{I}-\mathrm{A} / \mathrm{I}-\mathrm{E}^{1}$ & PerCP/cy5.5 & M5/114.15.12 & B253463 & Biolegend \\
\hline CD11a $^{1}$ & $\mathrm{PE}$ & M17/4 & E01070-82 & eBioScience \\
\hline Ly-6C & AF647 & ER-MP20 & 1113 & BioRAD \\
\hline Ly-6G & PE & $1 \mathrm{~A} 8$ & B221647 & Biolegend \\
\hline CD45 & APC/cy7 & $30-\mathrm{F} 11$ & B242535 & Biolegend \\
\hline Ly-6C & FITC & AL-21 & 4024747 & BDBiosciences \\
\hline CD45 & BV421 & $30-\mathrm{F} 11$ & B232006 & BioLegend \\
\hline CD206 (MMR) & AF647 & C068C2 & B230155 & BioLegend \\
\hline CX3CR1 & APC & SA011F11 & B200994 & BioLegend \\
\hline CX3CR1 & $\mathrm{PE}$ & SA011F11 & B194103 & BioLegend \\
\hline CD371 (CLEC12A) & $\mathrm{PE}$ & 5D3 & B211136 & BioLegend \\
\hline
\end{tabular}

${ }_{1}^{1}$ Antibodies used in high dimensional FACSymphony experiments 